\title{
EXPLORATORY X-RAY MONITORING OF LUMINOUS RADIO-QUIET QUASARS AT HIGH REDSHIFT: INITIAL RESULTS
}

\author{
Ohad Shemmer $^{1}$, W. N. Brandt ${ }^{2,3}$, Maurizio Paolillo $^{4,5}$, Shai Kaspi $^{6,7}$, Cristian Vignali ${ }^{8}$, \\ Matthew S. Stein ${ }^{1}$, Paulina Lira ${ }^{9}$, Donald P. Schneider ${ }^{2,3}$, and Robert R. Gibson ${ }^{10}$ \\ ${ }^{1}$ Department of Physics, University of North Texas, Denton, TX 76203, USA; ohad@unt.edu \\ ${ }^{2}$ Department of Astronomy \& Astrophysics, The Pennsylvania State University, University Park, PA 16802, USA \\ ${ }^{3}$ Institute for Gravitation and the Cosmos, The Pennsylvania State University, University Park, PA 16802, USA \\ ${ }^{4}$ Dipartimento di Scienze Fisiche, Università Federico II di Napoli, via Cinthia 6, I-80126 Napoli, Italy \\ ${ }^{5}$ ASI Science Data Center, Via del Politecnico snc, 00133 Rome, Italy \\ ${ }^{6}$ School of Physics \& Astronomy and the Wise Observatory, Tel Aviv University, Tel Aviv 69978, Israel \\ ${ }^{7}$ Department of Physics, Technion, Haifa 32000, Israel \\ ${ }^{8}$ Dipartimento di Astronomia, Università degli studi di Bologna, via Ranzani 1, I-40127 Bologna, Italy \\ ${ }^{9}$ Departamento de Astronomia, Universidad de Chile, Camino del Observatorio 1515, Santiago, Chile \\ ${ }^{10}$ Department of Astronomy, University of Washington, Box 351580, Seattle, WA 98195, USA \\ Received 2013 December 1; accepted 2014 January 17; published 2014 February 21
}

\begin{abstract}
We present initial results from an exploratory X-ray monitoring project of two groups of comparably luminous radio-quiet quasars (RQQs). The first consists of four sources at $4.10 \leqslant z \leqslant 4.35$, monitored by Chandra, and the second is a comparison sample of three sources at $1.33 \leqslant z \leqslant 2.74$, monitored by Swift. Together with archival $\mathrm{X}$-ray data, the total rest-frame temporal baseline spans $\sim 2-4 \mathrm{yr}$ and $\sim 5-13 \mathrm{yr}$ for the first and second group, respectively. Six of these sources show significant X-ray variability over rest-frame timescales of $\sim 10^{2}-10^{3}$ days; three of these also show significant $X$-ray variability on rest-frame timescales of $\sim 1-10$ days. The $X$-ray variability properties of our variable sources are similar to those exhibited by nearby and far less luminous active galactic nuclei (AGNs). While we do not directly detect a trend of increasing X-ray variability with redshift, we do confirm previous reports of luminous AGNs exhibiting X-ray variability above that expected from their luminosities, based on simplistic extrapolation from lower luminosity sources. This result may be attributed to luminous sources at the highest redshifts having relatively high accretion rates. Complementary UV-optical monitoring of our sources shows that variations in their optical-X-ray spectral energy distribution are dominated by the X-ray variations. We confirm previous reports of X-ray spectral variations in one of our sources, HS 1700+6416, but do not detect such variations in any of our other sources in spite of $X$-ray flux variations of up to a factor of $\sim 4$. This project is designed to provide a basic assessment of the X-ray variability properties of RQQs at the highest accessible redshifts that will serve as a benchmark for more systematic monitoring of such sources with future $\mathrm{X}$-ray missions.
\end{abstract}

Key words: galaxies: active - galaxies: nuclei - quasars: general - X-rays: galaxies

Online-only material: color figure

\section{INTRODUCTION}

Active galactic nuclei (AGNs) exhibit intensity fluctuations across the electromagnetic spectrum on timescales ranging from minutes to decades (e.g., Ulrich et al. 1997). While the source of these fluctuations is not yet fully understood, the bulk of the observed AGN variability is thought to arise in the accretion disk, corona, broad emission line region, and jet. Albeit its nonperiodic nature, AGN variability provides valuable information on the size and structure of the innermost regions of the central engine (e.g., Collier et al. 1998; Shemmer et al. 2001; Chartas et al. 2012). In particular, it can be used to estimate the masses of supermassive black holes (BHs; e.g., Kaspi et al. 2000b; Lu \& Yu 2001; Papadakis 2004; O'Neill et al. 2005; McHardy et al. 2006; Bentz et al. 2009; Ponti et al. 2012). Light-crossing time considerations imply that the timescales (amplitudes) of AGN flux variations should increase (decrease) with the emission-region size. Continuum variations in the X-rays, the bulk of which are thought to be emitted from the inner $\sim 10$ gravitational radii, are thus faster and stronger relative to those in, e.g., the optical band, and therefore provide a more efficient way of probing the central engines of AGNs, at least on relatively short timescales.

While X-ray variability in nearby AGNs has been the subject of intensive study (e.g., Nandra et al. 1997; Fiore et al. 1998;
Turner et al. 1999; Uttley et al. 2002; Markowitz et al. 2003), luminous quasars, found mostly at $z>1$ during the main growth phase of their supermassive BHs, have been relatively neglected until recently (see, e.g., Vagnetti et al. 2011; Gibson \& Brandt 2012). Being typically $\approx 10^{3}-10^{4}$ times more luminous than the highly variable local Seyfert galaxies, the emission regions of luminous quasars are expected to be physically larger and thus have slower and milder variations (e.g., Barr \& Mushotzky 1986; Green et al. 1993; Lawrence \& Papadakis 1993). Surprisingly, however, the past $\approx 15 \mathrm{yr}$ has seen tentative evidence suggesting evolution in the AGN X-ray variability properties, where sources of matched luminosity appear more $\mathrm{X}$-ray variable as redshift increases up to $z \sim 4$ (Almaini et al. 2000; Manners et al. 2002; Paolillo et al. 2004, hereafter P04). Almaini et al. (2000) found that $z>0.5$ sources in their ROSAT deep, flux-limited quasar sample did not show an anticorrelation between variability amplitude and luminosity as observed in local AGN. Using a large ROSAT sample of bright radio-quiet quasars (RQQs ${ }^{11}$ ) at $z \sim 2-4$, Manners et al. (2002) reported

\footnotetext{
11 Throughout this work we define radio-quiet AGNs as sources having $R=f_{v}(5 \mathrm{GHz}) / f_{v}(4400 \AA)<10$, following Kellermann et al. (1989). We also assume radio and optical-UV continua of the form $f_{v} \propto v^{-0.5}$ for computing $R$.
} 
Table 1

Basic X-Ray, Optical, and Radio Properties of the Chandra Sources

\begin{tabular}{|c|c|c|c|c|c|c|c|c|}
\hline Quasar & $\alpha(\mathrm{J} 2000.0)$ & $\delta(\mathrm{J} 2000.0)$ & $z$ & $\begin{array}{l}\text { Galactic } N_{\mathrm{H}}^{\mathrm{a}} \\
\left(10^{20} \mathrm{~cm}^{-2}\right)\end{array}$ & $\begin{array}{c}\log v L_{v}(2 \mathrm{keV})^{\mathrm{b}} \\
\left(\operatorname{erg~s}^{-1}\right)\end{array}$ & $\begin{array}{c}\log v L_{v}(2500 \AA)^{\mathrm{b}} \\
\left(\mathrm{erg} \mathrm{s}^{-1}\right)\end{array}$ & $\alpha_{\mathrm{OX}}^{\mathrm{c}}$ & $R^{\mathrm{d}}$ \\
\hline Q 0000-263 & 000322.9 & -260316.8 & 4.10 & 1.67 & 45.5 & 47.4 & -1.70 & $<4.7$ \\
\hline BR 0351-1034 & 035346.9 & -102519.0 & 4.35 & 4.08 & 45.2 & 46.9 & -1.69 & 1.2 \\
\hline PSS 0926+3055 & 092636.3 & +305505.0 & 4.19 & 1.89 & 45.7 & 47.7 & -1.76 & $<0.4$ \\
\hline PSS 1326+0743 & 132611.9 & +074358.4 & 4.17 & 2.01 & 45.5 & 47.5 & -1.76 & $<0.7$ \\
\hline
\end{tabular}

Notes.

a Obtained using the $N_{\mathrm{H}}$ tool at http://heasarc.gsfc.nasa.gov/cgi-bin/Tools/w3nh/w3nh.pl.

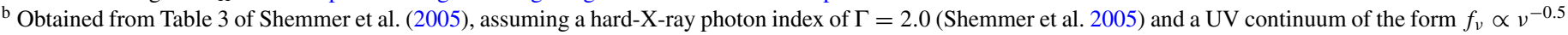
(Vanden Berk et al. 2001).

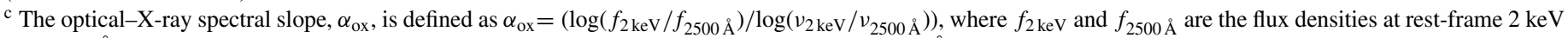
and $2500 \AA$, respectively. The $\alpha_{\text {ox }}$ values are obtained from the $\log v L_{v}(2 \mathrm{keV})$ and $\log v L_{v}(2500 \AA)$ data.

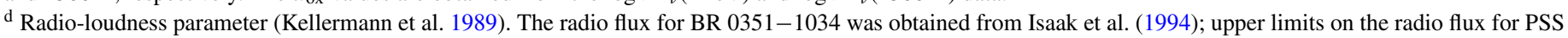
0926+3055 and PSS 1326+0743 were obtained from Becker et al. (1995), and from Condon et al. (1998) for Q 0000-263.

significant X-ray variations on rest-frame timescales as short as $\approx 1$ day. Utilizing the $1 \mathrm{Ms}$ Chandra Deep Field-South (CDF-S) survey, P04 found that at $2 \lesssim z \lesssim 3$ AGNs become more $\mathrm{X}$-ray variable on rest-frame timescales of $\sim 1-100$ days, and their variability amplitudes are larger than expected from extrapolations of their low-redshift counterparts. Significant $\mathrm{X}$-ray flux variations between two epochs separated by restframe timescales of $\sim 10-100$ days for sources at $z \sim 4-6$ were also reported by Shemmer et al. (2005). Collectively, these pieces of evidence suggest evolution of the X-ray variability mechanism, the X-ray emitting region size, or the accretion rate (e.g., Manners et al. 2002; P04).

However, evolutionary scenarios for these phenomena are perhaps puzzling given that the basic X-ray spectral properties of optically selected AGNs (i.e., hard-X-ray power-law photon index, intrinsic absorption, and the optical-X-ray spectral slope) have not evolved significantly over cosmic time up to $z \sim 6$ (e.g., Shemmer et al. 2005, 2006b; Vignali et al. 2005; Steffen et al. 2006; Just et al. 2007), consistent with findings from longer wavelengths (but see also Bechtold et al. 2003; Grupe et al. 2006; Kelly et al. 2007). X-ray monitoring of large numbers of highredshift quasars is therefore crucial for resolving this possible discrepancy and for testing evolutionary scenarios of the AGN central engine. It is particularly important to monitor quasars at $z>4$ regularly to add leverage at the highest accessible redshifts and to probe sources at the earliest stages of their formation. In spite of the fact that the number of X-ray-detected quasars at $z>4$ has grown more than tenfold since the 12 such sources known about a decade ago (Kaspi et al. 2000a), until recently only two X-ray epochs were available for a handful of such quasars (e.g., Shemmer et al. 2005; Gibson \& Brandt 2012) and, prior to this work, no systematic X-ray monitoring of such sources has been performed.

Quasar monitoring at the highest redshifts is challenging due to cosmic time dilation and the low flux levels of the sources involved (see, e.g., Kaspi et al. 2007). X-ray monitoring of such sources is particularly challenging since observations at higher redshifts necessarily involve harder X-rays, which are more difficult to detect given the steep quasar spectral energy distribution (SED) in the accessible energy range (Haardt \& Maraschi 1991, and references therein). Moreover, observations of such sources are currently feasible with only two observatories, Chandra $X$-Ray Observatory (hereafter Chandra; Weisskopf et al. 2000) and XMM-Newton (Jansen et al. 2001). Since the relatively faster variations in the $\mathrm{X}$-ray band compared to the optical-UV provide some compensation against cosmic time dilation, effective monitoring campaigns of high-redshift quasars may be performed within the typical lifetime of an X-ray observatory. We therefore embarked on an exploratory $\mathrm{X}$-ray monitoring project in order to extend our knowledge of quasar X-ray variability to the highest accessible redshifts while the current flagship X-ray observatories are operational.

This paper presents our monitoring strategy and the initial results of this project. We describe our target selection, the observations and their processing in Section 2, and present our basic findings in Section 3. Our results are discussed in Section 4 and briefly summarized in Section 5. Luminosity distances were computed using the standard cosmological model $\left(\Omega_{\Lambda}=0.7\right.$, $\Omega_{\mathrm{M}}=0.3$, and $H_{0}=70 \mathrm{~km} \mathrm{~s}^{-1} \mathrm{Mpc}^{-1}$; e.g., Spergel et al. 2007), and Galactic column densities were obtained from Dickey \& Lockman (1990).

\section{TARGET SELECTION, OBSERVATIONS, AND DATA REDUCTION}

\subsection{Strategy and Target Selection}

The aim of this exploratory monitoring project is to obtain, through time-series analyses, a qualitative assessment of the basic X-ray variability properties, such as amplitudes and timescales, for a small and carefully-selected sample of optically selected RQQs at high redshift and compare them with the $\mathrm{X}$-ray variability properties of AGNs at lower luminosities and redshifts. The selection of RQQs was intended to minimize interference of jet-related X-ray variations with those of the accretion-disk-corona system. The results of this project will guide more ambitious systematic X-ray observations of larger numbers of high-redshift sources with future X-ray missions. The ultimate goals are to identify the parameters that drive AGN X-ray variability and to test whether X-ray variability has evolved with cosmic time.

For our sample of RQQs at the highest accessible redshifts, we selected four sources at $4.10 \leqslant z \leqslant 4.35$ from Shemmer et al. (2005) that had at least two archival X-ray observations, per source, and were bright enough for economical Chandra observations (hereafter, the "Chandra sources"). These sources include Q 0000-263, BR 0351-1034, PSS 0926+3055, and PSS 1326+0743, all of which are optically selected quasars; their basic X-ray, optical, and radio properties appear in Table 1. Two of these sources, Q 0000-263 and BR 0351-1034, 
Table 2

Basic X-Ray, Optical, and Radio Properties of the Swift Sources

\begin{tabular}{|c|c|c|c|c|c|c|c|c|}
\hline Quasar & $\alpha(\mathrm{J} 2000.0)$ & $\delta(\mathrm{J} 2000.0)$ & $z$ & $\begin{array}{l}\text { Galactic } N_{\mathrm{H}}^{\mathrm{a}} \\
\left(10^{20} \mathrm{~cm}^{-2}\right)\end{array}$ & $\begin{array}{c}\log v L_{v}(2 \mathrm{keV})^{\mathrm{b}} \\
\left(\operatorname{erg~s}^{-1}\right)\end{array}$ & $\begin{array}{c}\log v L_{v}(2500 \AA) \\
\left(\operatorname{erg~s}^{-1}\right)\end{array}$ & $\alpha_{\mathrm{OX}}$ & $R^{\mathrm{e}}$ \\
\hline PG $1247+267$ & 125005.7 & +263107.6 & 2.04 & 0.90 & 45.7 & $47.4^{\mathrm{c}}$ & -1.69 & 0.4 \\
\hline PG $1634+706$ & 163429.0 & +703132.4 & 1.33 & 4.48 & 45.7 & $47.5^{\mathrm{c}}$ & -1.69 & 0.4 \\
\hline HS $1700+6416$ & 170100.6 & +64 1209.1 & 2.74 & 2.66 & 45.4 & $47.6^{\mathrm{d}}$ & -1.83 & $<0.1$ \\
\hline
\end{tabular}

Notes.

a Obtained using the $N_{\mathrm{H}}$ tool at http://heasarc.gsfc.nasa.gov/cgi-bin/Tools/w3nh/w3nh.pl.

${ }^{\mathrm{b}}$ Obtained from the mean X-ray flux of the Swift observations, assuming a hard-X-ray photon index of $\Gamma=2.0$ (see Section 2.3 ).

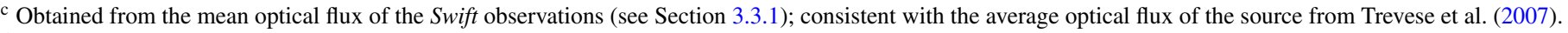

d Obtained from the mean optical flux of the Swift observations (see Section 3.3.1); consistent with the average optical flux of the source from Kaspi et al. (2007).

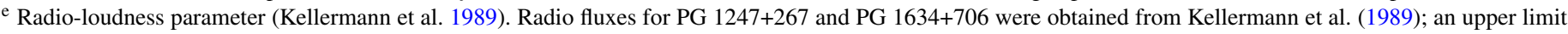
on the radio flux of HS 1700+6416 was obtained from Becker et al. (1995).

were discovered as part of the objective prism survey and the color survey with the Automatic Plate Measuring machine, respectively, using the UK Schmidt telescope in an effort to search for $z>4$ quasars (e.g., Webb et al. 1988; Irwin et al. 1991). The other two sources, PSS 0926+3055 and PSS 1326+0743, were discovered as $z>4$ quasars using multicolor selection with the Palomar Digital Sky Survey (e.g., Djorgovski et al. 1998). Beginning with Cycle 12, these sources are observed once per Chandra Cycle. The first X-ray epoch for each of these sources was obtained by shallow ROSAT (Truemper 1982) or Chandra observations. The second epoch for each of these was obtained by a lengthy XMM-Newton observation which also enabled searching for rapid X-ray variations on timescales of $\sim 1 \mathrm{hr}$ (see Shemmer et al. 2005, for more details about the first two epochs).

In order to distinguish between intrinsic and potential environmental effects on quasar variability it is necessary to break the strong luminosity-redshift dependence inherent in most quasar surveys. We therefore complemented the Chandra sources with a comparison sample of three RQQs with luminosities that are similar to those of the Chandra sample, but at considerably lower redshifts $(1.33 \leqslant z \leqslant 2.74)$. The sources of the comparison sample are sufficiently X-ray bright for monitoring with the Swift Gamma-Ray Burst Explorer (hereafter, the "Swift sources"; Gehrels et al. 2004). The Chandra sources, on the other hand, are too faint for Swift. The Swift sources were selected to have the largest number of archival $\mathrm{X}$-ray epochs without compromising the luminosity and redshift requirements. These sources include the optically selected quasars, PG 1247+267, PG 1634+706, and HS 1700+6416; their basic X-ray, optical, and radio properties appear in Table 2. The first two of these were discovered as part of the Bright Quasar Survey designed to identify UV-excess sources (e.g., Schmidt \& Green 1983). The third quasar, HS 1700+6416, was discovered in the course of the Hamburg wide-angle objective prism survey for bright quasars on the northern sky (Reimers et al. 1989).

The selection process of both samples, inevitably, introduces additional cross-calibration uncertainties stemming from the inclusion of data sets from different X-ray missions; these uncertainties are discussed further below. Nevertheless, our strategy maximally utilizes the X-ray archive and allows for the most efficient exploratory X-ray monitoring campaign of highredshift RQQs. Figure 1 shows the luminosities and redshifts of the sources in our two samples against the Sloan Digital Sky Survey (SDSS; York et al. 2000) quasar catalog of Schneider et al. (2010). Our two samples clearly mark the high-luminosity envelope of optically selected quasars and thereby probe X-ray

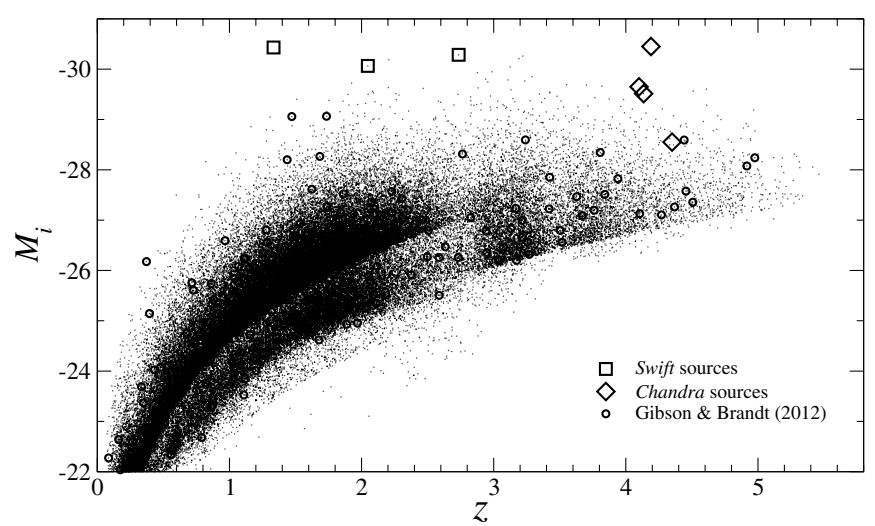

Figure 1. Absolute $i$-band magnitude vs. redshift for 105,783 SDSS quasars (dots; Schneider et al. 2010). The Chandra (Swift) sources are marked with diamonds (squares). SDSS quasars from the Gibson \& Brandt (2012) study are marked with circles. One Chandra source, PSS 1326+0743, and two Swift sources, PG 1247+267 and HS 1700+6416, are also SDSS quasars.

variability in a completely new region of the $L-z$ parameter space. Also shown in Figure 1 are the 264 SDSS quasars ${ }^{12}$ from Gibson \& Brandt (2012). Eight of their sources are at $z>4$, but none has more than two X-ray epochs. Our strategy is therefore complementary to the one used by Gibson \& Brandt (2012), and to the survey-based studies of, e.g., P04, Mateos et al. (2007), Vagnetti et al. (2011, 2013), Ponti et al. (2012), and Lanzuisi et al. (2014). Finally, both the Chandra and Swift sources are representative of highly luminous type 1 (i.e., unobscured) RQQs (with radio-loudness values almost an order of magnitude below the traditional $R=10$ cutoff). They all display typical blue continua with no broad absorption lines and typical emission-line properties (e.g., Green et al. 1980; Neugebauer et al. 1987; Baldwin et al. 1989; Schneider et al. 1989, 2010; Storrie-Lombardi et al. 1996; Vignali et al. 2003), hard-X-ray power-law photon indices in the range $\Gamma \sim 1.8-2.2$ (Page et al. 2004; Shemmer et al. 2005; Lanzuisi et al. 2012), and optical-Xray spectral slopes (hereafter $\alpha_{\text {ox }}$; see Table 1) consistent within $1 \sigma$ with expectations from their UV luminosities (Steffen et al. 2006; Just et al. 2007).

12 Two of these sources, SDSS J083454.89+553421.1 and SDSS J150407.51-024816.5, are AGNs which appear in an earlier version of the SDSS quasar catalog (Schneider et al. 2007), and were dropped from the Schneider et al. (2010) catalog since they did not meet the $M_{i}<-23$ criterion that would qualify them as quasars due to improved SDSS photometry. 
Table 3

Log of New Chandra Observations of the Chandra Sources

\begin{tabular}{lclcc}
\hline \hline Quasar & Cycle & Obs. Date & Obs. ID & $\begin{array}{c}\text { Exp. Time }^{\mathrm{a}} \\
(\mathrm{ks})\end{array}$ \\
\hline Q 0000-263 & 12 & 2011 Aug 29 & 12795 & 9.92 \\
& 13 & 2012 Sep 2 & 14215 & 9.93 \\
BR 0351-1034 & 12 & 2011 Sep 23 & 12796 & 9.92 \\
& 13 & 2011 Oct 28 & 14218 & 9.84 \\
PSS 0926+3055 & 12 & 2011 Mar 3 & 12793 & 4.98 \\
& 13 & 2012 Jan 13 & 14209 & 4.98 \\
PSS 1326+0743 & 12 & 2011 Mar 7 & 12794 & 4.98 \\
& 13 & 2012 Apr 30 & 14212 & 5.00 \\
\hline
\end{tabular}

Note. ${ }^{\text {a }}$ The Chandra exposure time has been corrected for detector dead time.

\subsection{New Chandra Observations}

We obtained Chandra snapshot observations of the Chandra sources in Cycles 12 and 13 (2011-2012); the observation log appears in Table 3. These data were obtained with the Advanced CCD Imaging Spectrometer (ACIS; Garmire et al. 2003) with the S3 CCD at the aimpoint using Faint mode for the event telemetry format in all the observations. Only events with grades of $0,2,3,4$, and 6 were considered in the analysis, which was performed using standard Chandra Interactive Analysis of Observations (CIAO) $)^{13}$ v4.1 routines. No background flares are present in these observations. The $\mathrm{X}$-ray counts in the observedframe ultrasoft band $(0.3-0.5 \mathrm{keV})$, soft band $(0.5-2 \mathrm{keV})$, hard band $(2-8 \mathrm{keV})$, and full band $(0.5-8 \mathrm{keV})$ were extracted with the WAVDETECT thread (Freeman et al. 2002) using wavelet transforms (with wavelet scale sizes of 1, 1.4, 2, 2.8, and 4 pixels) and a false-positive probability threshold of $10^{-3}$; these X-ray counts are reported in Table 4. The use of a relatively large false-positive probability threshold $\left(10^{-3}\right)$ is justified mainly due to the accurate a priori source positions and,

$13 \mathrm{http} / / / \mathrm{cxc} . c f a . h a r v a r d . e d u / c i a o /$ indeed, in most of the bands, source detection was also achieved with a more conservative false-positive probability threshold of $10^{-6}$. A manual inspection of all the Chandra images yielded consistent results with the WAVDETECT photometry. For each source, Table 4 also lists the band ratio, which is the ratio between the counts in the hard band and the soft band, the effective power-law photon index, ${ }^{14}$ the soft-band count rate, and the flux density at rest-frame $2 \mathrm{keV}$. Galactic absorptioncorrected fluxes in the observed-frame $0.5-2 \mathrm{keV}$ band were obtained using the Chandra PIMMS v4.4 tool, assuming a powerlaw model with $\Gamma=2.0$, and are discussed in Section 3.1.

\subsection{New Swift Observations}

We obtained Swift snapshot observations of the Swift sources in Cycles 3, 4, and 8 (2007-2013); the observation log appears in Table 5. The observations were obtained with the X-Ray Telescope (XRT; Burrows et al. 2005) simultaneously with the Ultraviolet Optical Telescope (UVOT; Roming et al. 2005) on board Swift. The UVOT filters used in each observation are given in Table 5; the analysis of the UVOT data is discussed in Section 3.3.1. The XRT Level 2 data were analyzed using standard XSELECT ${ }^{15}$ routines from FTOOLS (Blackburn 1995). For each source, counts in the observed-frame $0.2-10 \mathrm{keV}$ band were extracted from source regions with an aperture having a radius of 20 pixels (corresponding to $477^{\prime \prime 2}$ ) around the quasars' optical positions (this includes $90 \%$ of the energy of the point spread function at an observed-frame energy of $1.5 \mathrm{keV}$ ). Background counts were extracted from source-free regions that were six times larger (in area) than the source regions. The net counts are listed in Table 5. The corresponding X-ray fluxes were obtained using WebPIMMS,${ }^{16}$ corrected for Galactic absorption

\footnotetext{
14 The effective power-law photon index $\Gamma$, defined as $N(E) \propto E^{-\Gamma}$, was derived from the band ratio using the Chandra PIMMS v4.4 tool at http://cxc.harvard.edu/toolkit/pimms.jsp.

$15 \mathrm{http} / / /$ heasarc.gsfc.nasa.gov/docs/software/lheasoft/ftools/xselect/index. html

16 http://heasarc.nasa.gov/Tools/w3pimms.html
}

Table 4

Basic X-Ray Measurements from New Chandra Observations of the Chandra Sources

\begin{tabular}{|c|c|c|c|c|c|c|c|c|c|}
\hline \multirow[t]{2}{*}{ Quasar } & \multirow[t]{2}{*}{ Cycle } & \multicolumn{4}{|c|}{ Counts $^{\mathrm{a}}$} & \multirow[t]{2}{*}{ Band Ratio $^{b}$} & \multirow[t]{2}{*}{$\Gamma^{\mathrm{b}}$} & \multirow[t]{2}{*}{ Count Rate $^{c}$} & \multirow[t]{2}{*}{$f_{2 \mathrm{keV}}$} \\
\hline & & $0.3-0.5 \mathrm{keV}$ & $0.5-2 \mathrm{keV}$ & $2-8 \mathrm{keV}$ & $0.5-8 \mathrm{keV}$ & & & & \\
\hline Q 0000-263 & 12 & $4.0_{-1.9}^{+3.2}$ & $54.3_{-7.3}^{+8.4}$ & $14.8_{-3.8}^{+4.9}$ & $69.0_{-8.3}^{+9.4}$ & $0.27_{-0.08}^{+0.10}$ & $1.9 \pm 0.3$ & $5.47_{-0.74}^{+0.85}$ & 17.22 \\
\hline \multirow[t]{2}{*}{ BR 0351-1034 } & 12 & $<3.0$ & $11.8_{-3.4}^{+4.5}$ & $2.9_{-1.6}^{+2.9}$ & $14.7_{-3.8}^{+4.9}$ & $0.24_{-0.15}^{+0.26}$ & $2.1_{-0.7}^{+0.9}$ & $1.19_{-0.34}^{+0.46}$ & 4.20 \\
\hline & 13 & $<3.0$ & $9.8_{-3.1}^{+4.3}$ & $2.9_{-1.6}^{+2.9}$ & $12.7_{-3.5}^{+4.7}$ & $0.29_{-0.18}^{+0.32}$ & $1.9_{-0.7}^{+0.9}$ & $1.00_{-0.31}^{+0.43}$ & 3.52 \\
\hline PSS 0926+3055 & 12 & $2.0_{-1.3}^{+2.7}$ & $32.6_{-5.7}^{+6.8}$ & $10.8_{-3.2}^{+4.4}$ & $44.3_{-6.6}^{+7.7}$ & $0.33_{-0.11}^{+0.15}$ & $1.7_{-0.3}^{+0.4}$ & $6.53_{-1.14}^{+1.36}$ & 21.05 \\
\hline \multirow[t]{2}{*}{ PSS $1326+0743$} & 12 & $3.0_{-1.6}^{+2.9}$ & $33.7_{-5.8}^{+6.9}$ & $9.9_{-3.1}^{+4.3}$ & $43.5_{-6.6}^{+7.7}$ & $0.29_{-0.10}^{+0.14}$ & $1.9 \pm 0.4$ & $6.75_{-1.16}^{+1.34}$ & 21.75 \\
\hline & 13 & $2.0_{-1.3}^{+2.7}$ & $32.4_{-5.7}^{+6.8}$ & $11.9_{-3.4}^{+4.6}$ & $44.4_{-6.6}^{+7.7}$ & $0.37_{-0.12}^{+0.16}$ & $1.7_{-0.3}^{+0.4}$ & $6.49_{-1.13}^{+1.35}$ & 21.00 \\
\hline
\end{tabular}

Notes.

${ }^{a}$ Errors on the X-ray counts were computed according to Tables 1 and 2 of Gehrels (1986) and correspond to the $1 \sigma$ level; these were calculated using Poisson statistics. The upper limits are at the 95\% confidence level and were computed according to Kraft et al. (1991). Upper limits of 3.0 and 4.8 indicate that 0 and $1 \mathrm{X}$-ray counts, respectively, have been found within an extraction region of radius $1^{\prime \prime}$ centered on the optical position of the quasar (considering the background within this source-extraction region to be negligible).

${ }^{\mathrm{b}}$ We calculated errors at the $1 \sigma$ level for the band ratio (the ratio between the $2-8 \mathrm{keV}$ and $0.5-2 \mathrm{keV}$ counts) and effective photon index following the "numerical method" described in Section 1.7.3 of Lyons (1991); this avoids the failure of the standard approximate-variance formula when the number of counts is small (see Section 2.4.5 of Eadie et al. 1971). The photon indices have been obtained using Chandra PIMMS v4.4 which also implements the correction required to account for the ACIS quantum-efficiency decay at low energies (Townsley et al. 2000).

${ }^{c}$ Count rate computed in the observed-frame $0.5-2 \mathrm{keV}$ band in units of $10^{-3}$ counts s ${ }^{-1}$.

${ }^{\mathrm{d}}$ Flux density at rest-frame $2 \mathrm{keV}$ in units of $10^{-32} \mathrm{erg} \mathrm{cm}^{-2} \mathrm{~s}^{-1} \mathrm{~Hz}^{-1}$ assuming a power-law model with $\Gamma=2.0$. 
Table 5

Log of New Swift Observations of the Swift Sources

\begin{tabular}{|c|c|c|c|c|c|c|}
\hline Quasar & Obs. ID & Obs. Date & $\begin{array}{l}\text { Exp. Time } \\
\text { (ks) }\end{array}$ & $\begin{array}{l}\text { Net XRT } \\
\text { Counts }^{\mathrm{a}}\end{array}$ & $\begin{array}{l}\text { UVOT } \\
\text { Filters }\end{array}$ & $\operatorname{Bin}^{b}$ \\
\hline \multirow[t]{13}{*}{ PG $1247+267$} & 00036675001 & 2007 Jun 17 & 2.89 & $53.5_{-7.3}^{+8.4}$ & $U, W 2$ & 1 \\
\hline & 00036674001 & 2007 Jun 18 & 3.73 & $65.8_{-8.1}^{+9.2}$ & $M 2, W 2$ & 1 \\
\hline & 00090031001 & 2008 May 15 & 8.04 & $111.5_{-10.5}^{+11,6}$ & $V, U, B, W 1, M 2, W 2$ & 2 \\
\hline & 00090031002 & 2008 May 29 & 3.88 & $50.3_{-7.1}^{+8,1}$ & $V, U, B, W 1, M 2, W 2$ & 3 \\
\hline & 00090031003 & 2008 Nov 13 & 3.85 & $50.8_{-7.1}^{+8,2}$ & $V, U, B, W 1, M 2, W 2$ & 4 \\
\hline & 00036675002 & 2009 Feb 13 & 1.31 & $28.8_{-5.3}^{+6,4}$ & $U$ & 5 \\
\hline & 00036676001 & 2009 Feb 14 & 5.71 & $80.2_{-8.9}^{+10,0}$ & $U$ & 5 \\
\hline & 00091439001 & $2012 \operatorname{Dec} 8$ & 0.16 & $<4.8$ & $W 2$ & 6 \\
\hline & 00091439002 & 2013 Jan 31 & 1.93 & $20.7_{-4.5}^{+5,6}$ & $W 1$ & 6 \\
\hline & 00091439003 & 2013 Feb 4 & 0.15 & $<4.8$ & $W 1$ & 6 \\
\hline & 00091439004 & 2013 Mar 4 & 0.35 & $3.8_{-1.9}^{+3,1}$ & $W 1$ & 6 \\
\hline & 00091439005 & 2013 Mar 16 & 0.15 & $<4.8$ & $W 1$ & 6 \\
\hline & 00091439006 & 2013 Mar 23 & 0.55 & $10.3_{-3.2}^{+4,3}$ & $M 2$ & 6 \\
\hline \multirow[t]{16}{*}{ PG 1634+706 } & 00036672001 & 2007 Jun 29 & 1.32 & $33.2_{-5.7}^{+6,8}$ & $U$ & 1 \\
\hline & 00036673001 & 2007 Jun 29 & 1.48 & $44.8_{-6.7}^{+7,8}$ & $U$ & 1 \\
\hline & 00036672002 & 2007 Jul 11 & 7.34 & $196.5_{-14.0}^{+15,0}$ & $U$ & 2 \\
\hline & 00036671002 & 2008 Apr 22 & 2.09 & $94.3_{-9.7}^{+10,8}$ & $M 2$ & 3 \\
\hline & 00036673002 & 2008 Apr 24 & 2.56 & $96.2_{-9.8}^{+10,8}$ & $U$ & 3 \\
\hline & 00036671003 & 2008 Apr 26 & 3.00 & $134.8_{-11.6}^{+12,6}$ & $M 2$ & 4 \\
\hline & 00090030001 & 2008 May 15 & 3.72 & $161.0_{-12.7}^{+13,7}$ & $V, U, B, W 1, M 2, W 2$ & 5 \\
\hline & 00090030002 & 2008 Jun 12 & 3.25 & $92.3_{-9.6}^{+10,6}$ & $V, U, B, W 1, M 2, W 2$ & 6 \\
\hline & 00090030003 & 2009 Jan 18 & 2.75 & $71.3_{-8.4}^{+9,5}$ & $V, U, B, W 1, M 2, W 2$ & 7 \\
\hline & 00091438001 & 2012 Jun 11 & 1.26 & $43.7_{-6.6}^{+7,7}$ & $W 2$ & 8 \\
\hline & 00091438002 & 2012 Jun 18 & 0.37 & $17.3_{-4.1}^{+5,3}$ & $U$ & 8 \\
\hline & 00091438004 & 2012 Jun 22 & 0.42 & $18.3_{-4.2}^{+5,4}$ & $U, W 2$ & 8 \\
\hline & 00091438005 & 2012 Jun 25 & 0.17 & $7.7_{-2.7}^{+3,9}$ & $W 1$ & 8 \\
\hline & 00091438006 & 2012 Jun 26 & 0.36 & $23.3_{-4.8}^{+5,9}$ & $U$ & 8 \\
\hline & 00091438007 & 2012 Jun 28 & 0.50 & $19.8_{-4.4}^{+5,5}$ & $M 2$ & 8 \\
\hline & 00091438008 & 2012 Jul 1 & 5.88 & $348.5_{-18.7}^{+19,7}$ & $M 2, W 2$ & 9 \\
\hline \multirow[t]{23}{*}{ HS $1700+6416$} & 00036670001 & 2007 May 28 & 0.80 & $<3$ & $U$ & 1 \\
\hline & 00036670002 & 2007 Jun 7 & 5.44 & $17.2_{-4.1}^{+5.2}$ & $M 2$ & 1 \\
\hline & 00036669001 & 2007 Jun 27 & 4.92 & $6.0_{-2.4}^{+3.6}$ & $M 2$ & 1 \\
\hline & 00036668001 & 2007 Jun 27 & 4.34 & $10.8_{-3.2}^{+4.4}$ & $M 2$ & 2 \\
\hline & 00036669002 & 2008 Jan 17 & 2.10 & $11.7_{-3.4}^{+4.5}$ & $M 2$ & 2 \\
\hline & 00036668002 & 2008 Apr 24 & 2.21 & $14.8_{-3.8}^{+4.9}$ & $U$ & 2 \\
\hline & 00090032001 & 2008 May 16 & 10.31 & $33.8_{-5.8}^{+6.9}$ & $V, U, B, W 1, M 2, W 2$ & 3 \\
\hline & 00090032002 & 2008 May 30 & 4.64 & $20.7_{-4.5}^{+5.6}$ & $V, U, B, W 1, M 2, W 2$ & 4 \\
\hline & 00090032003 & 2008 May 31 & 4.22 & $19.3_{-4.4}^{+5.5}$ & $V, U, B, W 1, M 2, W 2$ & 4 \\
\hline & 00090032004 & 2009 Jan 21 & 1.99 & $6.2_{-2.4}^{+3.6}$ & $V, U, B, W 1, M 2, W 2$ & 5 \\
\hline & 00090032005 & 2009 Jan 22 & 4.32 & $12.2_{-3.4}^{+4.6}$ & $V, U, B, W 1, M 2, W 2$ & 5 \\
\hline & 00090032006 & 2009 Jan 29 & 0.50 & $<3$ & $V, U, B, W 1, M 2, W 2$ & 5 \\
\hline & 00090032007 & 2009 Jan 29 & 2.80 & $11.8_{-3.4}^{+4.5}$ & $V, U, B, W 1, M 2, W 2$ & 5 \\
\hline & 00091440001 & 2012 Jul 3 & 0.75 & $<8$ & $W 1$ & 6 \\
\hline & 00091440002 & 2012 Jul 5 & 0.88 & $3.7_{-1.8}^{+3.1}$ & $W 2$ & 6 \\
\hline & 00091440003 & 2012 Jul 6 & 4.15 & $19.3_{-4.4}^{+5.5}$ & $W 1, M 2$ & 6 \\
\hline & 00091440004 & 2012 Nov 23 & 0.82 & $6.3_{-2.4}^{+3.7}$ & $M 2$ & 6 \\
\hline & 00091440006 & 2012 Nov 28 & 1.82 & $4.8_{-2.1}^{+3.4}$ & $W 1$ & 6 \\
\hline & 00091440007 & $2012 \operatorname{Dec} 9$ & 1.47 & $6.2_{-2.4}^{+3.6}$ & $W 1, M 2$ & 7 \\
\hline & 00091440008 & $2012 \operatorname{Dec} 30$ & 1.39 & $4.5_{-2.0}^{+3.3}$ & $W 1$ & 7 \\
\hline & 00091440009 & 2013 Jan 5 & 0.72 & $<6.4$ & $W 2$ & 7 \\
\hline & 00091440010 & 2013 Jan 8 & 0.87 & $4.2_{-2.0}^{+3.2}$ & $U$ & 7 \\
\hline & 00091440011 & 2013 Jan 9 & 0.92 & $<8$ & $W 2$ & 7 \\
\hline
\end{tabular}


Table 5

(Continued)

\begin{tabular}{|c|c|c|c|c|c|c|}
\hline Quasar & Obs. ID & Obs. Date & $\begin{array}{l}\text { Exp. Time } \\
\text { (ks) }\end{array}$ & $\begin{array}{c}\text { Net XRT } \\
\text { Counts }^{\mathrm{a}}\end{array}$ & $\begin{array}{l}\text { UVOT } \\
\text { Filters }\end{array}$ & $\mathrm{Bin}^{\mathrm{b}}$ \\
\hline & 00091440012 & 2013 Jan 10 & 0.78 & $<8$ & $M 2$ & 7 \\
\hline & 00091440014 & 2013 Jan 13 & 3.55 & $17.5_{-4.1}^{+5.3}$ & $M 2, W 2$ & 8 \\
\hline & 00091440015 & 2013 Jan 15 & 1.50 & $4.7_{-2.1}^{+3.3}$ & $W 1$ & 8 \\
\hline & 00091440016 & 2013 Jan 16 & 0.80 & $5.8_{-2.3}^{+3.6}$ & $U$ & 8 \\
\hline
\end{tabular}

Notes.

${ }^{a}$ Errors on the X-ray counts were computed according to Tables 1 and 2 of Gehrels (1986) and correspond to the $1 \sigma$ level; these were calculated using Poisson statistics and include careful background subtraction. The upper limits are at the $95 \%$ confidence level and were computed according to Kraft et al. (1991). Upper limits of 3.0, 4.8, 6.4, and 8.0 indicate that $0,1,2$, and $3 \mathrm{X}$-ray counts, respectively, have been found within an extraction region of radius 20 pixels centered on the optical position of the quasar (assuming the background within this source-extraction region to be negligible).

b Swift observations having the same bin number were stacked in order to compute the X-ray variability probability and amplitude.

and assuming a power-law photon index of $\Gamma=2.0$, and are discussed in Section 3.1.

Ideally, we would have performed the X-ray time-series analyses in the same rest-frame energy band for all our sources. For the Chandra sources, we adopted the observed-frame 0.5-2.0 keV energy band, in which we obtain the largest fraction of the total counts from each source. Since the redshift of each Chandra source is $z \sim 4.2$, this band corresponds to the restframe $\sim 2.6-10.4 \mathrm{keV}$ energy band in each of these sources. We therefore repeated the Swift data analysis as described above but filtered the XRT event files to include only the rest-frame $\sim 2.6-10.4 \mathrm{keV}$ energy range for each Swift source. This filtering effectively reduced the number of counts by a factor of $\sim 2$ and reduced the signal-to-noise $(\mathrm{S} / \mathrm{N})$ level by $\sim 50 \%$ for each Swift observation. Using a Spearmanrank correlation we find that the X-ray fluxes obtained from the filtered observations are correlated with the corresponding fluxes in the observed-frame $0.2-10 \mathrm{keV}$ band at $>99.9 \%$ confidence level. This result is consistent with the results of P04 who did not find significant changes in their time-series analysis when considering a narrower observed-frame energy band that corresponded to the rest-frame energy band of most of their sources. We therefore perform time-series analyses using the observed-frame $0.2-10 \mathrm{keV}$ band for the Swift sources, thus maximizing the $\mathrm{S} / \mathrm{N}$ levels of their Swift observations.

\subsection{Archival X-Ray Data}

The data described in the previous two sections, for the Chandra and Swift sources, were complemented with archival observations from different X-ray missions. Specifically, Table 6 provides an observation log of the archival, i.e., non-Swift/XRT, X-ray data for the Swift sources. For PG 1634+706, the Einstein Imaging Proportional Counter (IPC) flux in the observedframe $0.28-3.29 \mathrm{keV}$ band was obtained from Tananbaum et al. (1986). Pointed ROSAT Position Sensitive Proportional Counters observations for the three Swift sources were analyzed according to the prescription from HEASARC ${ }^{17}$ using XSELECT and FTOOLS. We screened the data for "good" events in the observed-frame $0.5-2.0 \mathrm{keV}$ band, and extracted the counts from source regions having a radius of $50^{\prime \prime}$ (this includes $\sim 92 \%$ of the source flux at $1 \mathrm{keV}$ in the observed frame); background counts were obtained from source-free regions having radii of $150^{\prime \prime}$. The net count rates are consistent with

\footnotetext{
$\overline{17}$ http://heasarc.gsfc.nasa.gov/docs/rosat/ros_xselect_guide/\#tth_sEc4.1
}

those obtained from the ROSAT 2RXP catalog. ${ }^{18}$ For the ASCA observations of PG $1247+267$ and PG 1634+706, we obtained fluxes in the observed-frame $2-10 \mathrm{keV}$ band from George et al. (2000). The XMM-Newton observations of all three Swift sources were processed using standard XMM-Newton Science Analysis System $^{19}$ v6.5.0 tasks. For each source, counts were extracted from the European Photon Imaging Camera pn detector using an aperture with a $30^{\prime \prime}$ radius centered on the source (this includes $\sim 85 \%$ of the source flux at $1.5 \mathrm{keV}$ in the observed frame); background counts were extracted from apertures of similar sizes in nearby source-free regions. The Chandra observations of PG 1634+706 and HS 1700+6416 were analyzed in the same manner as described in Section 2.2 for the Chandra sources. Fluxes in the observed-frame $0.2-10 \mathrm{keV}$ band, corrected for Galactic absorption, for all these observations were obtained using WebPIMMS assuming a power-law photon index of $\Gamma=2.0$.

The archival X-ray data for the Chandra sources from the first two epochs were obtained from the literature as indicated in Table 7. Unabsorbed fluxes in the observed-frame $0.5-2.0 \mathrm{keV}$ band were determined from the fluxes provided in the literature using WebPIMMS and assuming a power-law photon index of $\Gamma=2.0$ (see also Shemmer et al. 2005).

\section{RESULTS}

\subsection{Variability Amplitudes}

Tables 7 and 8 provide the X-ray temporal data of the Chandra and Swift sources, respectively. The respective light curves are presented in Figures 2 and 3. The first step in the time-series analysis is to determine whether a source is variable. This is performed by applying a $\chi^{2}$ test to the light curve of each source, where the null hypothesis is that the flux in each epoch is consistent with the mean flux of the entire light curve, within the errors. This can be written as

$$
\chi^{2}=\frac{1}{N_{\text {obs }}-1} \sum_{i=1}^{N_{\text {obs }}} \frac{\left(f_{i}-\langle f\rangle\right)^{2}}{\sigma_{i}^{2}}
$$

where $f_{i}$ and $\sigma_{i}$ are the flux and its error in the $i$ th observation, respectively, $N_{\text {obs }}$ is the number of observations, and $\langle f\rangle$ is the mean flux of the light curve (e.g., P04). Table 9 gives the $\chi^{2}$

\footnotetext{
18 http://vizier.u-strasbg.fr/viz-bin/VizieR-3? - source=IX/30/2rxp

19 http://xmm.esac.esa.int/sas
} 
Table 6

Log of Archival Observations of the Swift Sources

\begin{tabular}{|c|c|c|c|c|c|}
\hline Quasar & Observatory & Obs. ID & Obs. Date & $\begin{array}{l}\text { Exp. Time } \\
\text { (ks) }\end{array}$ & References \\
\hline \multirow{3}{*}{ PG 1247+267 } & ROSAT & 701173 & 1993 Jan 4 & 2.10 & 1 \\
\hline & $A S C A$ & 73048000 & 1995 Jun 17 & 35.93 & 2 \\
\hline & XMM-Newton & 0143150201 & 2003 Jun 18 & 33.92 & 1,3 \\
\hline \multirow[t]{10}{*}{ PG 1634+706 } & Einstein & I05351 & 1981 Feb 6 & 1.83 & 4 \\
\hline & ROSAT & 700246 & 1991 Mar 15 & 9.01 & 1 \\
\hline & $A S C A$ & 71036000 & 1994 May 2 & 40.54 & 2 \\
\hline & Chandra & 1269 & 1999 Aug 21 & 10.83 & 1 \\
\hline & Chandra & 47 & 2000 Mar 23 & 5.39 & 1 \\
\hline & Chandra & 62 & 2000 Mar 23 & 4.85 & 1 \\
\hline & Chandra & 69 & 2000 Mar 24 & 4.86 & 1 \\
\hline & Chandra & 70 & 2000 Mar 24 & 4.86 & 1 \\
\hline & Chandra & 71 & 2000 Mar 24 & 4.41 & 1 \\
\hline & XMM-Newton & 0143150101 & 2002 Nov 22 & 19.71 & $1,3,5$ \\
\hline \multirow[t]{12}{*}{ HS $1700+6416$} & ROSAT & 701121 & 1992 Nov 13 & 15.50 & 1,6 \\
\hline & ROSAT & 701457 & 1993 Jul 21 & 26.32 & 1,6 \\
\hline & Chandra & 547 & 2000 Oct 31 & 49.53 & $1,7,8,9$ \\
\hline & XMM-Newton & 0107860301 & 2002 May 31 & 27.10 & $1,7,8,9$ \\
\hline & Chandra & 8032 & 2007 Nov 12 & 31.00 & 1,9 \\
\hline & Chandra & 9757 & 2007 Nov 13 & 20.78 & 1,9 \\
\hline & Chandra & 9756 & 2007 Nov 14 & 32.26 & 1,9 \\
\hline & Chandra & 9758 & 2007 Nov 16 & 23.37 & 1,9 \\
\hline & Chandra & 9759 & 2007 Nov 17 & 31.19 & 1,9 \\
\hline & Chandra & 9760 & 2007 Nov 19 & 16.95 & 1,9 \\
\hline & Chandra & 8033 & 2007 Nov 20 & 29.71 & 1,9 \\
\hline & Chandra & 9767 & 2007 Nov 21 & 9.04 & 1,9 \\
\hline
\end{tabular}

References. (1) This work; (2) George et al. 2000; (3) Page et al. 2004; (4) Tananbaum et al. 1986; (5) Piconcelli et al. 2005; (6) Reimers et al. 2005; (7) Just et al. 2007; (8) Misawa et al. 2008; (9) Lanzuisi et al. 2012.

Table 7

X-Ray Light Curve Data for the Chandra Sources

\begin{tabular}{lcccc}
\hline \hline Quasar & JD & $f_{\mathrm{x}}{ }^{\text {a }}$ & Observatory & Reference \\
\hline Q 0000-263 & 2448587.3 & $30.0 \pm 3.8$ & ROSAT & $1,2,3$ \\
& 2452450.8 & $12.6 \pm 0.7$ & XMM-Newton & $3,4,5$ \\
& 2455802.8 & $22.6_{-3.1}^{+3.5}$ & Chandra & 6 \\
& 2456173.4 & $18.7_{-2.8}^{+3.2}$ & Chandra & 6 \\
BR 0351-1034 & 2448649.1 & $57.0 \pm 12.5$ & ROSAT & 2,3 \\
& 2453036.0 & $11.7 \pm 2.2$ & XMM-Newton & $3,5,7$ \\
& 2455827.5 & $5.3_{-1.5}^{+2.0}$ & Chandra & 6 \\
PSS 0926+3055 & 2455863.1 & $4.4_{-1.4}^{+1.9}$ & Chandra & 6 \\
& 2452344.6 & $27.7_{-4.7}^{+4.7}$ & Chandra & 3,8 \\
& 2453322.5 & $39.0_{-2.5}^{+2.6}$ & XMM-Newton & 3 \\
& 2455624.4 & $27.2_{-4.7}^{+5.7}$ & Chandra & 6 \\
PSS 1326+0743 & 2455939.7 & $19.2_{-4.2}^{+4.6}$ & Chandra & 6 \\
& 2452285.3 & $24.4_{-3.3}^{+4.3}$ & Chandra & 3,8 \\
& 2453002.0 & $27.9_{-2.5}^{+1.9}$ & XMM-Newton & 3 \\
& 2455628.1 & $28.2_{-4.8}^{+5.8}$ & Chandra & 6 \\
& 2456048.0 & $27.2_{-4.8}^{+5.7}$ & Chandra & 6 \\
\hline
\end{tabular}

Notes. ${ }^{a}$ Galactic absorption-corrected flux in the observed-frame $0.5-2 \mathrm{keV}$ band in units of $10^{-15} \mathrm{erg} \mathrm{cm}^{-2} \mathrm{~s}^{-1}$.

References. (1) Bechtold et al. 1994; (2) Kaspi et al. 2000a; (3) Shemmer et al. 2005; (4) Ferrero \& Brinkmann 2003; (5) Grupe et al. 2006; (6) This work; (7) Grupe et al. 2004; (8) Vignali et al. 2003.

values as well as the corresponding degrees of freedom (dof; i.e., $\left.N_{\text {obs }}-1\right)$ and the $\chi^{2}$ distribution probability by which the null hypothesis can be rejected $(p)$.
Due to Swift's planning and scheduling constraints, our requested exposures of the Swift sources were, in most cases, divided into shorter exposure segments with uneven integration times as can be seen in Table 5. For the purpose of determining whether a Swift source is variable and to compute its variability amplitude, we stacked the counts in the Swift exposures into separate bins as indicated in Table 5. By attempting to match our original requested exposures, we binned the Swift data by stacking exposures, chronologically, up to a minimum of $4 \mathrm{ks}$, $3 \mathrm{ks}$, and $8 \mathrm{ks}$, rounded to the nearest ks, for PG $1247+267$, PG 1634+706, and HS 1700+6416, respectively. However, we stacked exposures from Cycle 8 separately from the other Cycles due to the large time gap up to Cycle 8 . The original requested exposures were designed to provide uncertainties of $\approx 10 \%-20 \%$ on the flux measurements of the Swift sources. The $\chi^{2}$ values and probabilities for the Swift sources shown in Table 9 were computed based on their binned Swift data.

Since the light curves of our sources are composed of data from different X-ray observatories, flux uncertainties stemming from instrument cross calibrations may affect the measured variability properties. One particular concern is that the two Chandra sources that have three Chandra observations and one observation with XMM-Newton appear to be either non-variable (PSS 1326+0743) or much less variable (PSS 0926+3055) than the other two Chandra sources that have two observations with Chandra, one with ROSAT, and one with XMM-Newton. This trend may be a consequence of real larger variations due to the longer temporal baselines introduced by the ROSAT observations (as discussed further below), or due to the fact that data from multiple observatories introduce additional crosscalibration uncertainties. Sources may thus appear more variable 
Table 8

X-Ray Light Curve Data for the Swift Sources

\begin{tabular}{|c|c|c|c|c|}
\hline Quasar & $\mathrm{JD}$ & $f_{\mathrm{x}}^{\mathrm{a}}$ & $\begin{array}{l}\text { Observatory } \\
\text { Code }\end{array}$ & $\alpha_{\mathrm{ox}}$ \\
\hline \multirow[t]{13}{*}{ PG $1247+267$} & 2448991.5 & $11.5 \pm 1.4$ & 1 & $\ldots$ \\
\hline & 2449886.0 & $7.1 \pm 0.5$ & 2 & $\ldots$ \\
\hline & 2452808.9 & $3.5 \pm 0.2$ & 3 & $\ldots$ \\
\hline & 2454268.5 & $6.7_{-0.9}^{+1.0}$ & 4 & -1.65 \\
\hline & 2454269.8 & $6.4_{-0.8}^{+0.9}$ & 4 & -1.64 \\
\hline & 2454601.5 & $5.0 \pm 0.5$ & 4 & -1.70 \\
\hline & 2454615.9 & $4.7_{-0.7}^{+0.8}$ & 4 & -1.72 \\
\hline & 2454783.7 & $4.8_{-0.7}^{+0.8}$ & 4 & -1.71 \\
\hline & 2454876.5 & $8.0_{-1.5}^{+1.8}$ & 4 & -1.64 \\
\hline & 2454877.1 & $5.1 \pm 0.6$ & 4 & -1.72 \\
\hline & 2456323.5 & $3.9_{-0.8}^{+1.1}$ & 4 & -1.75 \\
\hline & 2456356.5 & $4.0_{-1.9}^{+3.3}$ & 4 & -1.75 \\
\hline & 2456375.1 & $6.8_{-2.1}^{+2.9}$ & 4 & -1.66 \\
\hline \multirow[t]{26}{*}{ PG 1634+706 } & 2444641.5 & $16.0 \pm 3.2$ & 5 & $\ldots$ \\
\hline & 2448330.9 & $15.4 \pm 0.1$ & 1 & $\ldots$ \\
\hline & 2449475.3 & $25.4 \pm 1.0$ & 2 & $\ldots$ \\
\hline & 2451412.5 & $15.4 \pm 0.3$ & 6 & $\cdots$ \\
\hline & 2451627.4 & $23.3 \pm 0.6$ & 6 & $\ldots$ \\
\hline & 2451627.5 & $23.4 \pm 0.6$ & 6 & $\cdots$ \\
\hline & 2451627.5 & $22.9 \pm 0.6$ & 6 & $\cdots$ \\
\hline & 2451627.6 & $22.1 \pm 0.6$ & 6 & $\ldots$ \\
\hline & 2451627.7 & $23.5_{-0.6}^{+0.7}$ & 6 & $\cdots$ \\
\hline & 2452601.4 & $28.5 \pm 1.0$ & 3 & $\ldots$ \\
\hline & 2454280.5 & $11.1_{-1.9}^{+2.3}$ & 4 & -1.78 \\
\hline & 2454280.9 & $13.5_{-2.0}^{+2.3}$ & 4 & -1.75 \\
\hline & 2454292.5 & $11.9_{-0.8}^{+0.9}$ & 4 & -1.77 \\
\hline & 2454579.3 & $20.1_{-2.1}^{+2.3}$ & 4 & -1.68 \\
\hline & 2454581.0 & $16.7_{-1.7}^{+1.9}$ & 4 & -1.71 \\
\hline & 2454582.6 & $20.0_{-1.7}^{+1.9}$ & 4 & -1.68 \\
\hline & 2454601.9 & $19.2_{-1.5}^{+1.6}$ & 4 & -1.69 \\
\hline & 2454629.5 & $12.6_{-1.3}^{+1.5}$ & 4 & -1.76 \\
\hline & 2454850.1 & $11.5_{-1.4}^{+1.5}$ & 4 & -1.77 \\
\hline & 2456090.0 & $15.4_{-2.3}^{+2.7}$ & 4 & -1.68 \\
\hline & 2456096.8 & $20.7_{-4.9}^{+6.3}$ & 4 & -1.65 \\
\hline & 2456100.8 & $19.2_{-4.4}^{+5.6}$ & 4 & -1.64 \\
\hline & 2456103.9 & $20.0_{-7.0}^{+10.2}$ & 4 & -1.65 \\
\hline & 2456104.6 & $23.1_{-4.8}^{+5.9}$ & 4 & -1.64 \\
\hline & 2456107.4 & $17.7_{-3.9}^{+4.9}$ & 4 & -1.66 \\
\hline & 2456110.2 & $26.3_{-1.4}^{+1.5}$ & 4 & -1.60 \\
\hline \multirow[t]{19}{*}{ HS $1700+6416$} & 2448939.5 & $2.3 \pm 0.2$ & 1 & $\ldots$ \\
\hline & 2449189.5 & $1.8 \pm 0.2$ & 1 & $\cdots$ \\
\hline & 2451849.5 & $0.70 \pm 0.04$ & 6 & $\cdots$ \\
\hline & 2452426.3 & $2.5_{-0.7}^{+0.5}$ & 3 & $\cdots$ \\
\hline & 2454258.7 & $1.3_{-0.3}^{+0.4}$ & 4 & -1.89 \\
\hline & 2454278.6 & $0.5_{-0.2}^{+0.3}$ & 4 & -2.04 \\
\hline & 2454279.1 & $1.0_{-0.3}^{+0.4}$ & 4 & -1.92 \\
\hline & 2454416.6 & $0.8 \pm 0.1$ & 6 & $\ldots$ \\
\hline & 2454418.2 & $0.7 \pm 0.1$ & 6 & $\cdots$ \\
\hline & 2454419.3 & $0.8 \pm 0.1$ & 6 & $\cdots$ \\
\hline & 2454420.9 & $1.0 \pm 0.1$ & 6 & $\cdots$ \\
\hline & 2454421.9 & $0.8 \pm 0.1$ & 6 & $\cdots$ \\
\hline & 2454423.6 & $1.1 \pm 0.1$ & 6 & $\cdots$ \\
\hline & 2454424.6 & $0.9 \pm 0.1$ & 6 & $\cdots$ \\
\hline & 2454425.9 & $0.8 \pm 0.1$ & 6 & $\ldots$ \\
\hline & 2454482.7 & $2.3_{-0.7}^{+0.9}$ & 4 & -1.79 \\
\hline & 2454580.5 & $2.7_{-0.7}^{+0.9}$ & 4 & -1.75 \\
\hline & 2454602.5 & $1.3_{-0.2}^{+0.3}$ & 4 & -1.88 \\
\hline & 2454617.0 & $1.8_{-0.4}^{+0.5}$ & 4 & -1.82 \\
\hline
\end{tabular}

Table 8

(Continued)

\begin{tabular}{|c|c|c|c|c|}
\hline Quasar & JD & $f_{\mathrm{x}}^{\mathrm{a}}$ & $\begin{array}{c}\text { Observatory } \\
\text { Code }\end{array}$ & $\alpha_{\mathrm{Ox}}$ \\
\hline & 2454618.0 & $1.9_{-0.4}^{+0.5}$ & 4 & -1.82 \\
\hline & 2454852.5 & $1.3_{-0.5}^{+0.7}$ & 4 & -1.88 \\
\hline & 2454853.5 & $1.2_{-0.3}^{+0.4}$ & 4 & -1.89 \\
\hline & 2454861.5 & $1.7_{-0.5}^{+0.7}$ & 4 & -1.81 \\
\hline & 2456113.5 & $1.7_{-0.8}^{+1.4}$ & 4 & -1.84 \\
\hline & 2456115.1 & $1.9_{-0.4}^{+0.5}$ & 4 & -1.82 \\
\hline & 2456254.6 & $3.1_{-1.2}^{+1.8}$ & 4 & -1.72 \\
\hline & 2456259.6 & $1.1_{-0.5}^{+0.8}$ & 4 & -1.91 \\
\hline & 2456271.3 & $1.7_{-0.7}^{+1.0}$ & 4 & -1.83 \\
\hline & 2456291.6 & $1.3_{-0.6}^{+1.0}$ & 4 & -1.87 \\
\hline & 2456300.6 & $2.0_{-0.9}^{+1.5}$ & 4 & -1.81 \\
\hline & 2456304.7 & $2.1_{-0.5}^{+0.6}$ & 4 & -1.80 \\
\hline & 2456305.8 & $2.0_{-0.5}^{+0.6}$ & 4 & -1.83 \\
\hline & 2456307.8 & $1.3_{-0.6}^{+0.9}$ & 4 & -1.89 \\
\hline & 2456308.5 & $3.0_{-1.2}^{+1.8}$ & 4 & -1.74 \\
\hline
\end{tabular}

Notes. Observatory Codes: (1) ROSAT; (2) ASCA; (3) XMM-Newton; (4) Swift; (5) Einstein; (6) Chandra.

${ }^{a}$ Galactic absorption-corrected flux in the observed-frame $0.2-10 \mathrm{keV}$ band in units of $10^{-13} \mathrm{erg} \mathrm{cm}^{-2} \mathrm{~s}^{-1}$.

if these uncertainties are not properly taken into account. It underscores the importance of conducting any monitoring project in a uniform way with the same instrument in the same band in order to obtain the most reliable results.

The relative uncertainties in the cross calibrations among the X-ray observatories used in this work (except for Einstein/IPC) are typically of the order of $\lesssim 10 \%$ (e.g., Kirsch et al. 2005). After adding, in quadrature, an uncertainty of $10 \%$ to the error bar on each flux value to account for these uncertainties (following Saez et al. 2012), the significance of the $\chi^{2}$ test drops below $90 \%$ for each of the Swift sources and for PSS $0926+3055$. For the Swift sources, this effect is mainly due to the fact that the $\mathrm{S} / \mathrm{N}$ of their Swift data are lower, on average, than those of the other X-ray data and their sampling pattern is more complex, relying heavily on the shortest sampling timescales where uncertainties on the variability power are larger; as discussed below, the variability amplitude increases with sampling timescale.

To overcome the sampling bias, and to allow a better comparison with the temporal patterns of the Chandra sources, we computed the $\chi^{2}$ value for each Swift source, including the additional cross-calibration uncertainties of $10 \%$, and using a light curve composed of one data point per X-ray observatory, where the fluxes from each observatory were averaged (resulting in a substantial increase in the S/N of the Swift data). As a result, PG 1247+267 and HS 1700+6416 are variable with $>90 \%$ confidence, whereas PG $1634+706$ shows variability with $>90 \%$ confidence only when the first (i.e., Einstein) and last (i.e., Swift) data points are excluded. The removal of these two points was done in order to match, as closely as possible, the temporal sampling of the other Swift and Chandra sources, and due to the fact that the cross-calibration uncertainty of the Einstein/IPC with respect to the other instruments we use has not yet been determined (Kirsch et al. 2005). In particular, the rest-frame time separations between the Einstein epoch and the ROSAT epoch (i.e., second epoch) as well as between the mean epoch of the Swift 


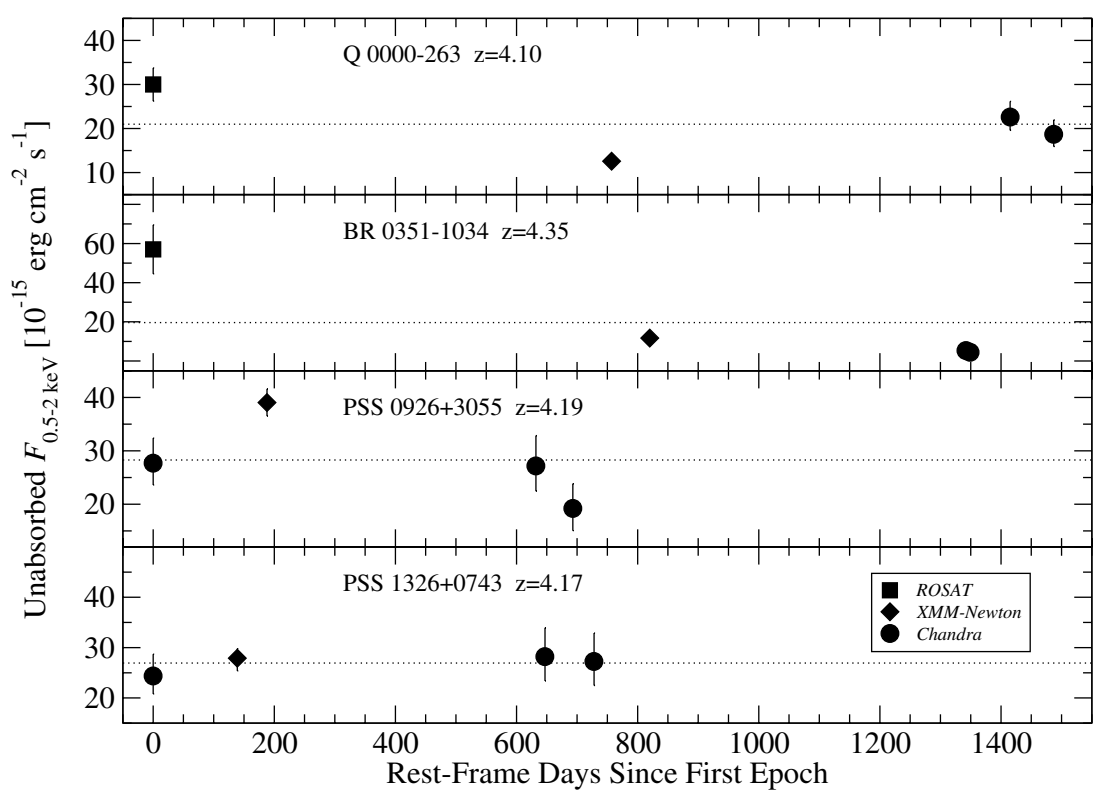

Figure 2. X-ray light curves of the Chandra sources. Galactic-absorption corrected flux in the observed-frame $0.5-2 \mathrm{keV}$ band is plotted as a function of rest-frame time (in days) relative to the first X-ray epoch for each source. Squares, diamonds, and circles mark ROSAT, XMM-Newton, and Chandra observations, respectively. The dotted line in each panel indicates the mean flux.

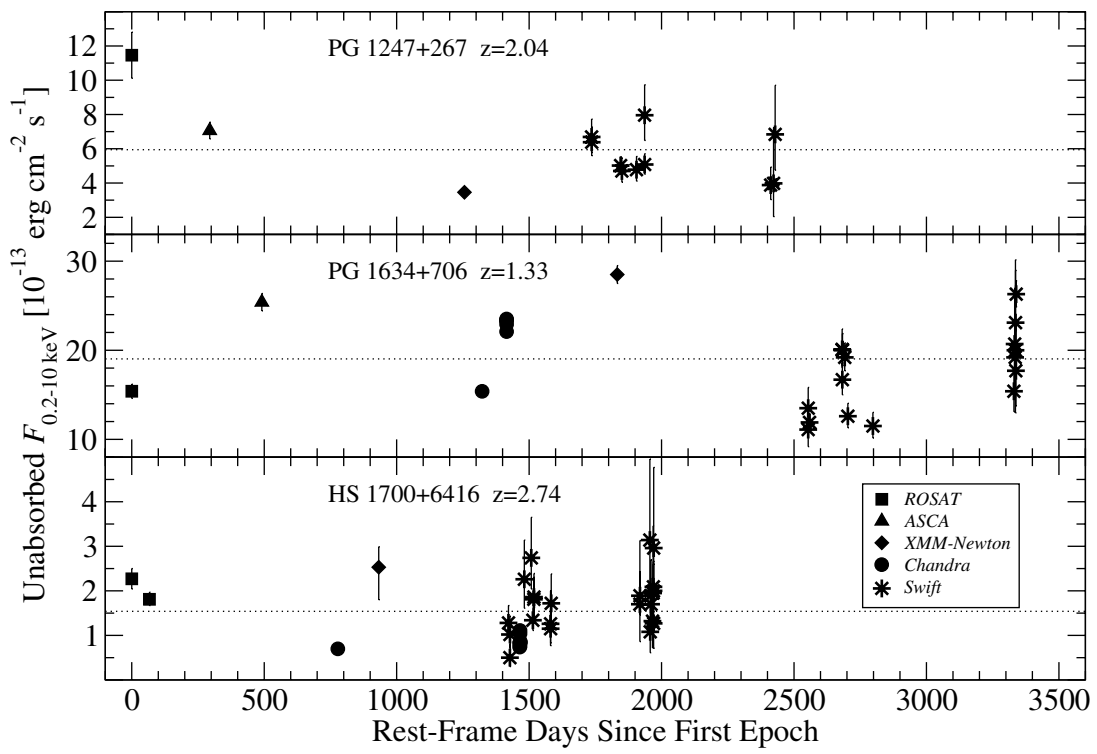

Figure 3. X-ray light curves of the Swift sources. Galactic-absorption corrected flux in the observed-frame $0.2-10 \mathrm{keV}$ band is plotted as a function of rest-frame time (in days) relative to the first X-ray epoch for each source. Squares, triangles, diamonds, circles, and stars indicate ROSAT, ASCA, XMM-Newton, Chandra, and Swift observations, respectively. The much earlier Einstein observation of PG $1634+706$ with an unabsorbed flux of $F_{0.2-10 \mathrm{keV}}=(16.0 \pm 3.2) \times 10^{-13} \mathrm{erg} \mathrm{cm}^{-2} \mathrm{~s}^{-1}$ is not shown in the middle panel, for clarity. The dotted line in each panel indicates the mean flux.

observations and the XMM-Newton epoch (i.e., fifth epoch) are a factor of $\sim 2-3$ larger than the average and median rest-frame time separations between adjacent epochs in all our sources.

The light curve of PSS $0926+3055$ is composed of three Chandra epochs and one XMM-Newton epoch, and the relative calibration uncertainties between Chandra and XMM-Newton are considerably lower than $10 \%$ (e.g., Snowden 2002). We therefore maintain that small cross-calibration uncertainties do not warrant an artificial increase in the flux uncertainties for this source. In fact, when the added cross-calibration uncertainty is $3 \%$, the $\chi^{2}$ significance is $>90 \%$. To summarize, we consider all our sources, except for PSS $1326+0743$, to be X-ray variable at $>90 \%$ confidence.
The X-ray variability amplitude is computed as the source excess variance defined by Turner et al. (1999; see also Nandra et al. 1997) as

$$
\sigma_{\mathrm{rms}}^{2}=\frac{1}{N_{\mathrm{obs}}\langle f\rangle^{2}} \sum_{i=1}^{N_{\mathrm{obs}}}\left[\left(f_{i}-\langle f\rangle\right)^{2}-\sigma_{i}^{2}\right],
$$

who also define the error on $\sigma_{\mathrm{rms}}^{2}$ as $s_{D} /\left(\langle f\rangle^{2} \sqrt{N_{\mathrm{obs}}}\right)$, where $s_{D}$ is given by

$$
s_{D}^{2}=\frac{1}{N_{\mathrm{obs}}-1} \sum_{i=1}^{N_{\mathrm{obs}}}\left\{\left[\left(f_{i}-\langle f\rangle\right)^{2}-\sigma_{i}^{2}\right]-\sigma_{\mathrm{rms}}^{2}\langle f\rangle^{2}\right\}^{2} .
$$


Table 9

X-Ray Variability Indicators

\begin{tabular}{llccr}
\hline \hline Sample & \multicolumn{1}{c}{ Quasar } & $\chi^{2}$ (dof) & $1-p^{\mathrm{a}}$ & \multicolumn{1}{c}{$\sigma_{\text {rms }}^{2}$} \\
\hline Chandra & Q 0000-263 & $46.4(3)$ & $4.8 \times 10^{-10}$ & $0.07 \pm 0.05$ \\
& BR 0351-1034 & $58.6(3)$ & $1.2 \times 10^{-12}$ & $1.13 \pm 0.71$ \\
& PSS 0926+3055 & $7.3(3)$ & $6.2 \times 10^{-2}$ & $0.04 \pm 0.04$ \\
& PSS 1326+0743 & $0.2(3)$ & $9.7 \times 10^{-1}$ & $-0.02 \pm 0.01$ \\
Swift & PG 1247+267 & $21.7(8)$ & $5.5 \times 10^{-3}$ & $0.14 \pm 0.09$ \\
& PG 1634+706 & $35.7(18)$ & $7.8 \times 10^{-3}$ & $0.07 \pm 0.02$ \\
& HS 1700+6416 & $34.8(19)$ & $1.5 \times 10^{-2}$ & $0.14 \pm 0.04$
\end{tabular}

Note. ${ }^{\text {a }}$ The probability $p$ of the $\chi^{2}$ distribution, given the $\chi^{2}$ value and the degrees of freedom (dof).

The $\sigma_{\text {rms }}^{2}$ values and their errors for our sources are listed in Table 9; for the Swift sources, these were computed using the binned Swift observations. The above expression for the error on $\sigma_{\text {rms }}^{2}$, however, represents only a "formal" error which does not take into account the scatter intrinsic to any red-noise random process, as discussed in Vaughan et al. (2003) and Allevato et al. (2013). The quantity $\sigma_{\text {rms }}^{2}$ is also prone to systematic effects and biases stemming from the duration of the monitoring and from the power-law slope of the AGN power spectral density (PSD) function. The intrinsic $\sigma_{\mathrm{rms}}^{2}$ tends to increase with the monitoring duration (e.g., Vagnetti et al. 2011) due to the red-noise nature of the PSD function, while the observed $\sigma_{\text {rms }}^{2}$ increases with steeper PSD power-law slopes with respect to the intrinsic value due to power leakage from lower frequencies (Allevato et al. 2013).

\subsubsection{Comparison with the CDF-S 2 Ms Survey}

We compare the variability amplitudes of our sources with those of X-ray-selected AGNs from the 2 Ms exposure of the CDF-S survey (Luo et al. 2008). This sample forms an extension of the P04 variability study based on the $1 \mathrm{Ms}$ exposure of the CDF-S and is a subset of the investigation based on its $4 \mathrm{Ms}$ exposure (M. Paolillo et al., in preparation). The comparison with the data from the $2 \mathrm{Ms}$ exposure, instead of the P04 data or with the full $4 \mathrm{Ms}$ exposure, is motivated by the fact that the timescales probed in this survey, spanning $\sim 2-7 \mathrm{yr}$ in the rest frame, closely match the timescales probed for our Chandra and Swift sources. A distribution of the rest-frame timescales probed for sources in the Luo et al. (2008) CDF-S survey is shown in Figure 4. The analysis of the CDF-S data was performed following the steps outlined in P04. The current study includes 81 point sources with $L_{0.5-8 \mathrm{keV}}>10^{42} \mathrm{erg} \mathrm{s}^{-1}$ and with $>300$ counts in order to meet the requirement of having $\sim 10$ counts, on average, per Chandra observation. We further removed 20 of these sources that have radio detections (Xue et al. 2011) in order to minimize potential jet-related variability. We do not imply, however, that all the remaining sources are formally radio quiet or that no RQQs were culled in this process; a rigorous analysis of the radio and optical properties of the CDF-S sources is beyond the scope of this work. Of the remaining 61 sources, 13 are deemed non variable, as their $\chi^{2}$ values indicate variability with $<90 \%$ confidence; yet, these non-variable sources are taken into account in the analysis below in order to prevent biasing of the variability-amplitude distribution (see, e.g., P04).

In Figure 5 we plot the variability amplitudes of our sources as a function of X-ray luminosity and show for reference the $61 \mathrm{CDF}-\mathrm{S}$ sources (cf. Figure 12 of P04). To match the $\mathrm{X}$-ray luminosities of the CDF-S sources, the X-ray luminosities of our sources were extrapolated to the observed-frame

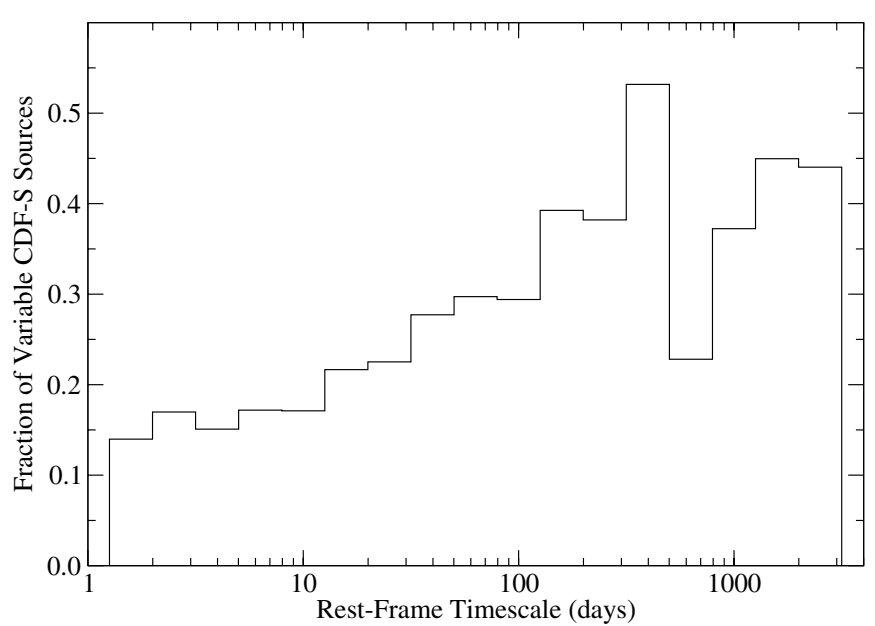

Figure 4. Distribution of rest-frame timescales probed in the $2 \mathrm{Ms}$ exposure of the CDF-S. These timescales are similar to those probed for our Chandra and Swift sources.

$0.5-8 \mathrm{keV}$ band by assuming a photon index of $\Gamma=2.0$ for each source in this band. Our sources extend the CDF-S parameter space by $\Delta z \simeq 1$ and by an order of magnitude in X-ray luminosity. Figure 5 also shows simulated variability amplitudes as a function of luminosity which are described in Section 4. By inspection of Figure 5, one can see that extremely luminous AGNs at the highest accessible redshifts continue to display $\mathrm{X}$-ray variability amplitudes that are as high or even higher than those of many of their lower-luminosity counterparts. We do not see a trend of decreasing variability amplitude with increasing luminosity as might have been expected based on studies of nearby AGNs (see Section 1). Our findings therefore bolster the results of $\mathrm{P} 04$ and support previous tentative reports of increased X-ray variability in luminous AGNs. However, as we discuss below, we do not find clear evidence that the X-ray variability amplitude depends on redshift.

\subsection{Variability Timescales}

Although the Chandra sources lack sufficient observations for a quantitative temporal analysis, we detect significant $\mathrm{X}$-ray variability over characteristic rest-frame timescales of $\approx 10^{2}-10^{3}$ days in the variable sources. No X-ray variability is detected in these sources over rest-frame timescales of $\lesssim 0.1$ days (Shemmer et al. 2005). Additional Chandra observations are required for a quantitative analysis, especially on intermediate rest-frame timescales ( $\approx 1-10$ days). The Swift sources, on the other hand, have sufficient observations for a more detailed investigation of X-ray variability timescales using variability structure functions (SFs). Even though the SF is not as sensitive to the variability power at each timescale as the PSD function (e.g., Emmanoulopoulos et al. 2010), it is the best analysis tool available for sparsely sampled light curves with few epochs (e.g., Vagnetti et al. 2011).

We computed the SF, i.e., $\Delta m$, for each Swift source following the definition from Fiore et al. (1998):

$$
\Delta m_{j i}=\left|2.5 \log \left[f\left(t_{j}\right) / f\left(t_{i}\right)\right]\right|
$$

where $f\left(t_{j}\right)$ and $f\left(t_{i}\right)$ are the fluxes of the source at epochs $t_{j}$ and $t_{i}$, respectively, such that $t_{j}>t_{i}$, and every $t_{i}$ is measured 


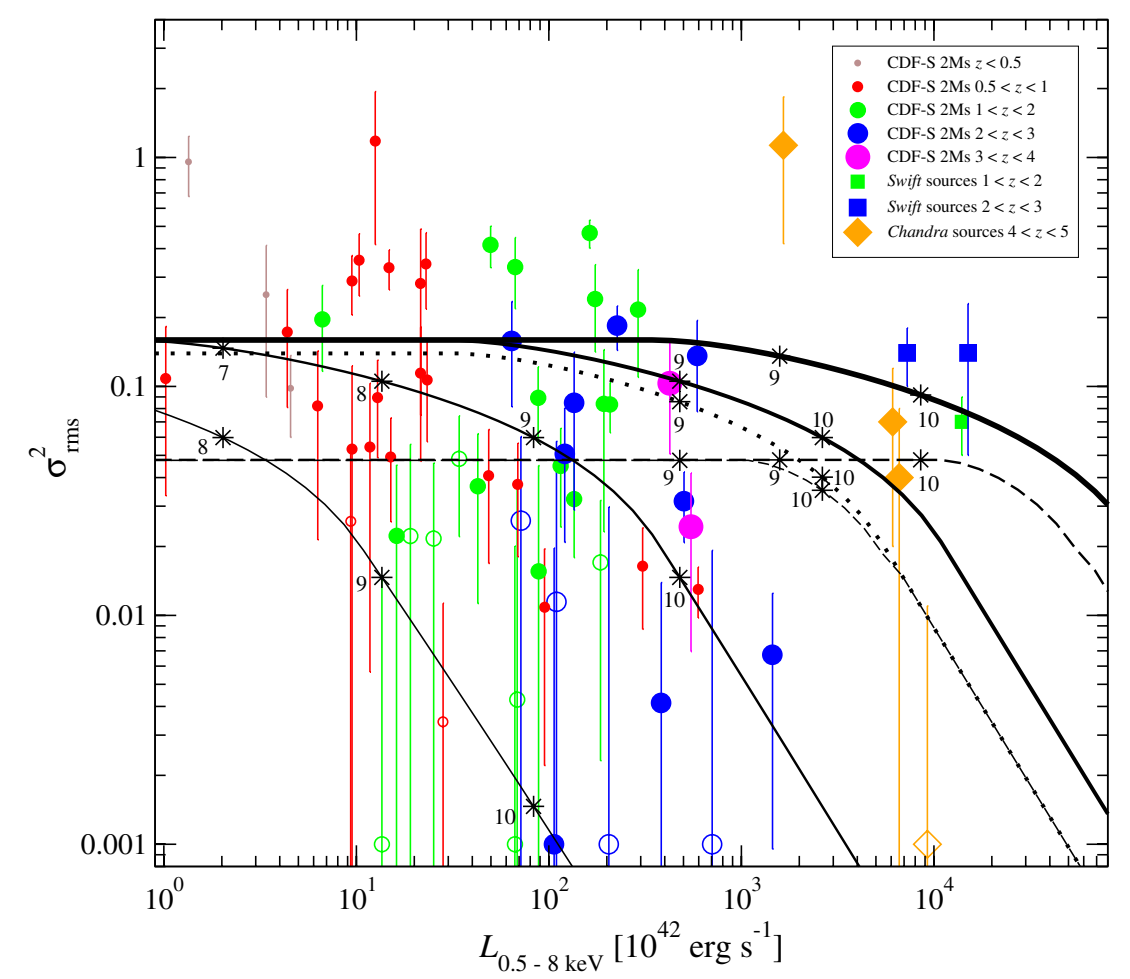

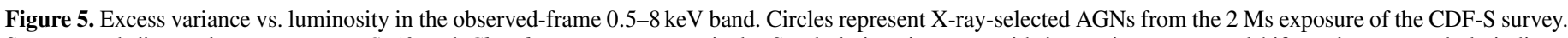

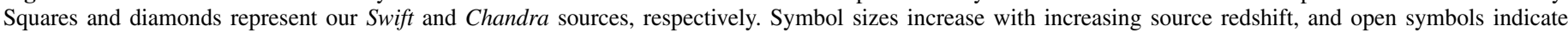

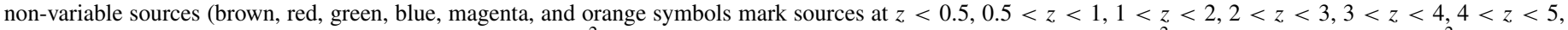

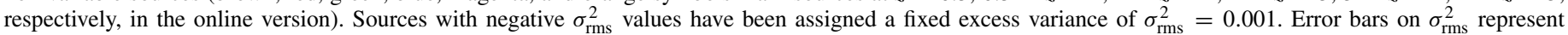

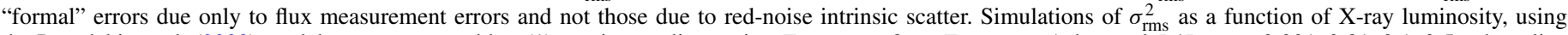

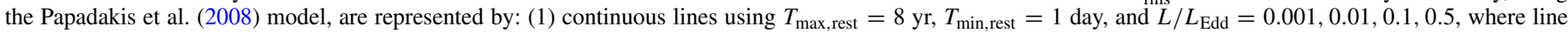

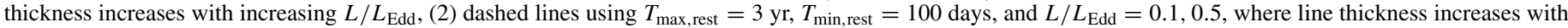

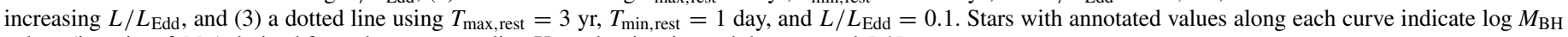
values (in units of $M_{\odot}$ ) derived from the corresponding X-ray luminosity and the assumed $L / L_{\text {Edd }}$.

(A color version of this figure is available in the online journal.)

in rest-frame days since the first epoch (i.e., $t_{1}=0$ ). We binned the SF of each source into 13 rest-frame time bins forming a geometric sequence of the form $\left(\Delta t_{\text {rest }}\right)_{n}=2^{n-1}$ days where $n \in 1, \cdots, 13$, and computed the mean SF, $\langle\Delta m\rangle$, for each of these time bins.

Figure 6 shows the mean SFs of our Swift sources. The SFs show a general trend of increasing variability on longer rest-frame timescales ( $\gtrsim 100$ days), although variability at some level is detected in all the Swift sources at much shorter rest-frame timescales, down to $\sim 1$ day, in particular, in HS 1700+6416 (see Table 8). However, at the shortest timescale, the SF may be dominated by the measurement noise (e.g., Hughes et al. 1992). We also computed an ensemble SF including all the Swift sources, by averaging the $\Delta m$ values of all the sources in each rest-frame time bin. This ensemble SF is plotted in Figure 7 against the ensemble SFs of steepand flat-X-ray-spectrum quasars from Fiore et al. (1998) that have luminosities that are almost two orders of magnitude lower than those of the Swift sources. The ensemble SF of the Swift sources appears to be remarkably similar to the ensemble SF of the steep-X-ray spectrum quasars, which also have higher accretion rates (Fiore et al. 1998), at least on the shorter timescales $(\lesssim 10$ days). Figure 7 also shows that additional X-ray monitoring is required to characterize better the ensemble SF on rest-frame timescales of $\sim 10-100$ days and to compare with the behaviors of the X-ray variability SFs from Vagnetti et al. (2011). Finally, we searched for rapid X-ray variability, on timescales of a few minutes in the rest frame, in the Swift sources by binning in time each Swift observation with more than 100 source counts. We did not find evidence for such rapid variability in the only two sources that have more than this number of counts in a single observation, PG $1247+267$ and PG 1634+706 (see also Lanzuisi et al. 2012 for the lack of rapid variability, over timescales of minutes, in the Chandra observations of HS 1700+6416).

\subsection{SED Variability}

AGNs often display complex connections between X-ray variability and variations in the fluxes of other spectral bands due to the nature of the corresponding emission regions and the different PSD functions involved (see, e.g., Uttley \& McHardy 2004, and references therein). We search for connections between the X-ray and optical-UV variations of our sources by obtaining optical-UV observations that are simultaneous (using Swift/UVOT) or nearly simultaneous (using ground-based observations) with the X-ray observations. Specifically, the optical-UV data allow searching for variations in $\alpha_{\mathrm{ox}}$ (defined in Table 1), considered to be a measure of the accretion-disk corona reprocessing of optical-UV radiation emitted from the accretion disk. Significant $\alpha_{\mathrm{ox}}$ variations can probe the interplay between the accretion disk and the corona and provide insight 


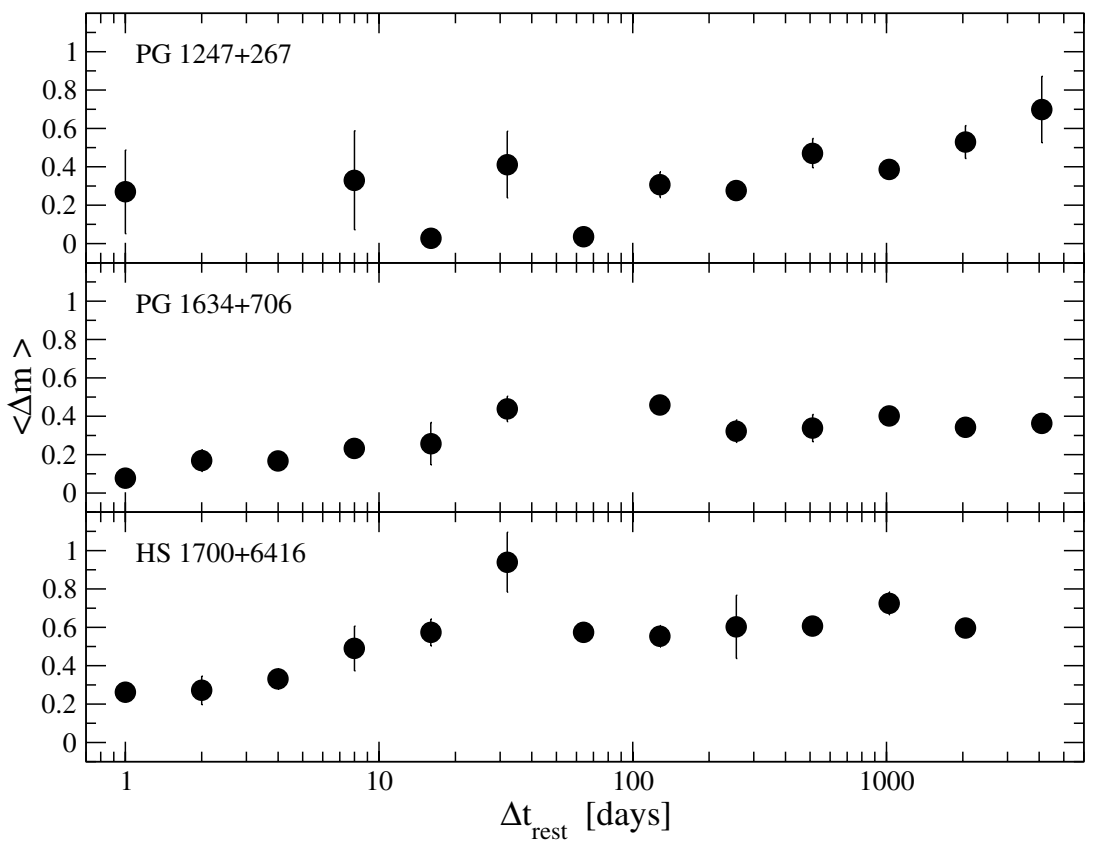

Figure 6. Mean SFs of the Swift sources. Average magnitude difference in each time bin is plotted as a function of rest-frame time interval. These sources are more variable on longer timescales than on shorter ones, and significant $\mathrm{X}$-ray variability is detected on rest-frame timescales as short as $\sim 1$ day.

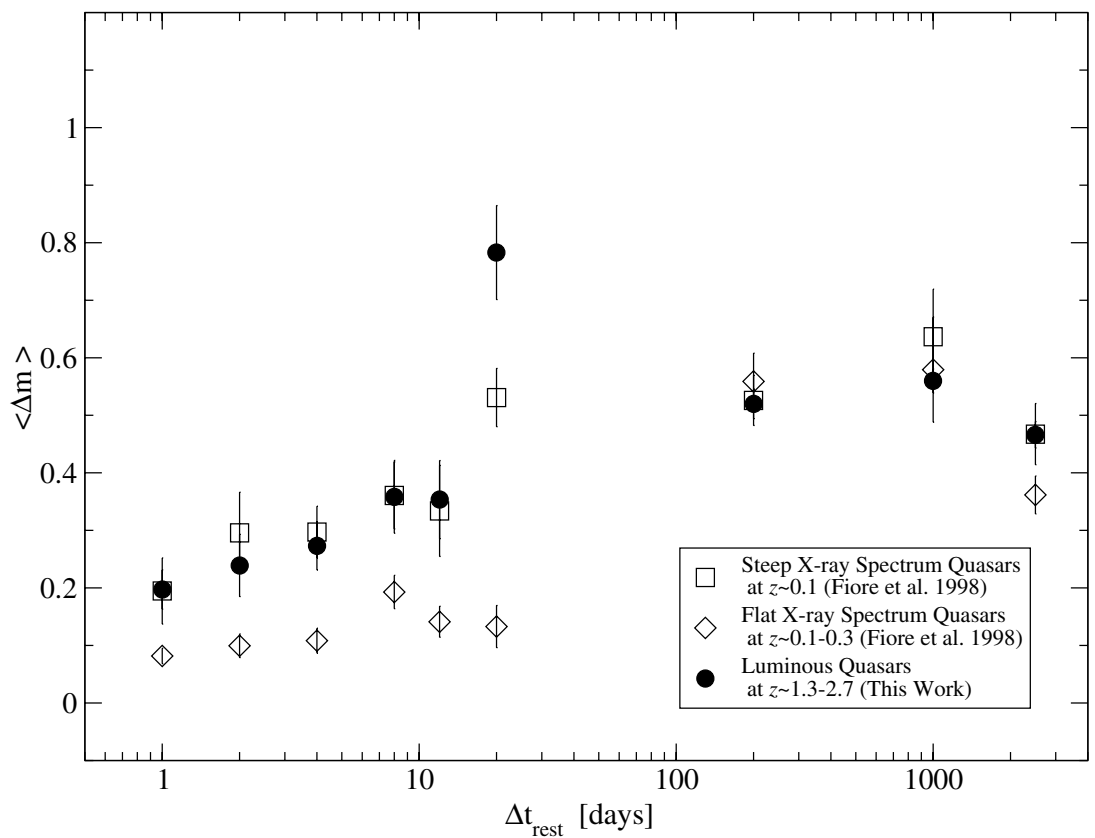

Figure 7. Ensemble SF of the Swift sources (circles) compared to the ensemble SFs of the steep- and flat-X-ray-spectrum quasars at low redshift, marked by squares and diamonds, respectively, adapted from Fiore et al. (1998). Units are similar to those in Figure 6. The temporal behavior of the Swift sources is remarkably similar to that of steep-X-ray-spectrum quasars, perhaps due to the fact that both groups have similarly high accretion rates.

into the accretion processes in the high-redshift sources under study.

\subsubsection{Swift/UVOT Observations}

The UVOT data for the Swift sources were extracted from the Italian Space Agency Science Data Center ${ }^{20}$ archive. Flux densities in each UVOT band were obtained by employing standard HEASOFT ${ }^{21}$ tasks on the UVOT sky images. The UVOT filters available for each Swift observation are listed in Table 5.

\footnotetext{
${ }^{20} \mathrm{http}: / /$ www.asdc.asi.it/mmia/index.php?mission=swiftmastr

$21 \mathrm{http}: / /$ heasarc.gsfc.nasa.gov/docs/software/lheasoft/
}

For each Swift source, the flux density at a rest-frame wavelength of $2500 \AA, f_{v}(2500 \AA)$, was obtained by extrapolating the flux density available for the band with an effective wavelength which is closest to $2500 \AA$ using a power-law continuum of the form $f_{v} \propto v^{-0.5}$ (Vanden Berk et al. 2001). These bands were either $V, B$, or $U$; hence the rest-frame effective wavelength for each observation, from which the $f_{v}(2500 \AA)$ value was extrapolated, was typically $\gtrsim 1200 \AA$ A. For observations lacking $V, B$, and $U$ filters, the flux densities from one of the other three available UV filters were converted to $V$-band fluxes using the mean flux-density ratio between that filter and the $V$ filter, based on available observations of the same source that included both 
Table 10

Optical Photometry

\begin{tabular}{|c|c|c|c|c|c|c|c|c|c|c|}
\hline Quasar & Observatory & $\begin{array}{l}\text { Obs. } \\
\text { Date }\end{array}$ & $\begin{array}{c}g^{\prime} \\
(\mathrm{mag})\end{array}$ & $\begin{array}{c}r^{\prime} \\
(\mathrm{mag})\end{array}$ & $\begin{array}{c}i^{\prime} \\
(\mathrm{mag})\end{array}$ & $\begin{array}{c}z^{\prime} \\
(\mathrm{mag})\end{array}$ & $\begin{array}{c}B \\
(\mathrm{mag})\end{array}$ & $\begin{array}{c}V \\
(\mathrm{mag})\end{array}$ & $\begin{array}{c}R \\
(\mathrm{mag})\end{array}$ & $\begin{array}{c}I \\
(\mathrm{mag})\end{array}$ \\
\hline \multirow[t]{3}{*}{ Q 0000-263 } & WO & 2011 Sep 4 & $18.93 \pm 0.02$ & $17.45 \pm 0.02$ & $\ldots$ & $\cdots$ & $19.58 \pm 0.04$ & $18.23 \pm 0.02$ & $17.16 \pm 0.02$ & $\cdots$ \\
\hline & WO & 2012 Sep 14 & $18.93 \pm 0.03$ & $17.48 \pm 0.01$ & $\cdots$ & $\cdots$ & $19.45 \pm 0.09$ & $18.28 \pm 0.02$ & $17.18 \pm 0.03$ & $\cdots$ \\
\hline & WO & 2012 Sep 15 & $18.97 \pm 0.02$ & $17.48 \pm 0.01$ & $\cdots$ & $\cdots$ & $19.53 \pm 0.04$ & $18.26 \pm 0.02$ & $17.17 \pm 0.01$ & $\cdots$ \\
\hline \multirow[t]{4}{*}{ BR 0351-1034 } & WO & 2011 Mar 3 & $\ldots$ & $19.39 \pm 0.06$ & $\ldots$ & $\cdots$ & $\ldots$ & $\ldots$ & $19.24 \pm 0.05$ & $\cdots$ \\
\hline & WO & 2011 Mar 5 & $\cdots$ & $19.33 \pm 0.04$ & $\cdots$ & $\cdots$ & $\cdots$ & $\ldots$ & $\ldots$ & $\cdots$ \\
\hline & WO & 2011 Sep 26 & $\ldots$ & $19.33 \pm 0.03$ & $\ldots$ & $\cdots$ & $\ldots$ & $20.59 \pm 0.09$ & $19.29 \pm 0.04$ & $\cdots$ \\
\hline & LCO & 2011 Oct 29 & $\ldots$ & $\ldots$ & $\ldots$ & $\ldots$ & $22.79 \pm 0.11$ & $20.55 \pm 0.02$ & $19.35 \pm 0.03$ & $\ldots$ \\
\hline \multirow[t]{2}{*}{ PSS 0926+3055 } & WO & 2011 Mar 4 & $18.45 \pm 0.01$ & $17.13 \pm 0.01$ & $17.01 \pm 0.01$ & $17.22 \pm 0.03$ & $\ldots$ & $17.83 \pm 0.02$ & $16.90 \pm 0.01$ & $16.60 \pm 0.02$ \\
\hline & WO & 2012 Feb 4 & $18.55 \pm 0.05$ & $17.35 \pm 0.11$ & $17.19 \pm 0.06$ & $\ldots$ & $\cdots$ & $17.94 \pm 0.05$ & $17.11 \pm 0.08$ & $16.66 \pm 0.04$ \\
\hline \multirow[t]{3}{*}{ PSS $1326+0743$} & WO & 2011 Mar 8 & $19.15 \pm 0.10$ & $\ldots$ & $\ldots$ & $\ldots$ & $\cdots$ & $18.47 \pm 0.03$ & $17.48 \pm 0.02$ & $16.88 \pm 0.03$ \\
\hline & WO & 2011 Mar 14 & $19.28 \pm 0.03$ & $17.82 \pm 0.10$ & $17.51 \pm 0.10$ & $17.15 \pm 0.03$ & $\cdots$ & $18.47 \pm 0.02$ & $17.49 \pm 0.02$ & $16.77 \pm 0.12$ \\
\hline & WO & 2012 May 1 & $\ldots$ & $17.79 \pm 0.06$ & $17.61 \pm 0.07$ & $\ldots$ & $\cdots$ & $18.52 \pm 0.14$ & $17.59 \pm 0.07$ & $16.69 \pm 0.09$ \\
\hline
\end{tabular}

the UV filter and the $V$ filter. The resulting $\alpha_{\text {ox }}$ values are given in Table 8.

The largest differences in $\alpha_{\mathrm{ox}}$ between two Swift observations are $\Delta \alpha_{\mathrm{ox}}=0.11,0.18$, and 0.32 for PG $1247+267$, PG $1634+706$, and HS 1700+6416, respectively; however, only for PG 1634+706 is this difference statistically significant (at $>3 \sigma$ ). For all three Swift sources, the differences in $\alpha_{\text {ox }}$ are dominated by the X-ray variations (as shown in Table 8). At least for PG $1634+706$, these variations in $\alpha_{\mathrm{ox}}$ are larger than the $1 \sigma$ uncertainty on $\alpha_{\text {ox }}$ values measured from the correlation between $\alpha_{\text {ox }}$ and the optical-UV luminosity for such high-luminosity AGNs (Steffen et al. 2006). This result indicates that X-ray variability may provide a significant contribution to the dispersion in the luminosity-corrected $\alpha_{\text {ox }}$ values of luminous AGNs. Our results are consistent with the findings of Vagnetti et al. (2013) for a low-redshift AGN sample observed with Swift (see also Gibson et al. 2008; Vagnetti et al. 2010; Gibson \& Brandt 2012). Although a detailed optical-UV-X-ray cross-correlation analysis is beyond the scope of this work, the dominance of the X-ray variations in the variations of $\alpha_{\mathrm{ox}}$ is also manifested by the considerably lower optical amplitudes of all three Swift sources during periods that overlap with our monitoring. The amplitudes of the optical continuum variations of these sources are normally within $\sim 10 \%$ (see Kaspi et al. 2007; Trevese et al. 2007), while the typical X-ray amplitudes are much larger, up to factors of a few.

\subsubsection{Ground-based Photometry}

Optical imaging of the Chandra sources was performed with the $1 \mathrm{~m}$ telescope at the Tel Aviv University Wise Observatory (WO). Images were obtained with the PI CCD camera which has a $13^{\prime} \times 13^{\prime}$ field of view with a scale of $0^{\prime} .58 \mathrm{pix}^{-1}$. The sources were observed with a combination of several of the $g^{\prime}, r^{\prime}, i^{\prime}, z^{\prime}$ filters (see Fukugita et al. 1996) and Johnson $B, V, R, I$ filters which were available on particular nights of observations. The images were reduced in the standard way using IRAF ${ }^{22}$ routines. Broad-band light curves for the quasars were produced by comparing their instrumental magnitudes to those of nonvariable stars in the field (see, e.g., Netzer et al. 1996, for more details). The quoted uncertainties on the photometric measurements include the fluctuations due to photon statistics and the scatter in the measurement of the non-variable stars

\footnotetext{
22 IRAF (Image Reduction and Analysis Facility) is distributed by the National Optical Astronomy Observatory, which is operated by AURA, Inc., under cooperative agreement with the National Science Foundation.
}

used. Flux calibration was done using the fluxes of a few tens of field stars from known catalogs. For PSS 0926+3055 and PSS $1326+0743$ we used the SDSS catalog in order to flux calibrate the images in the $g^{\prime}, r^{\prime}, i^{\prime}, z^{\prime}$ filters. To derive the flux for the field stars in the $V, R, I$ bands we used the transformations between SDSS magnitudes and $V, R, I$ magnitudes as given by R. Lupton. ${ }^{23}$ For Q 0000-263 and BR 0351-1034 we used the USNO-A2.0 catalog and the prescription given by B. Gary. ${ }^{24}$ We derived the $B, V, R$ magnitudes of the field stars and used the transformations between SDSS and $B, V, R$ magnitudes of Jester et al. (2005) to derive the flux in the $g^{\prime}$ and $r^{\prime}$ filters. The flux calibration performed here is only an approximation, due to the calibration prescriptions used, and there might be systematic uncertainties in the flux calibration on the order of $\sim 0.5 \mathrm{mag}$. These uncertainties are not included in the uncertainties quoted on the measurements below since we are interested only in the relative flux changes between the observed epochs.

We also performed optical imaging of BR 0351-1034 with the du Pont $2.5 \mathrm{~m}$ telescope at Las Campanas Observatory (LCO). Images were obtained in the Johnson $B, V, R$ bands with the Wide Field CCD camera which has a scale of 0.484 pix $^{-1}$ and is equipped with a WF4K detector. ${ }^{25}$ The images were reduced and calibrated in the same manner as described above for the WO data. The observation log from the WO and LCO as well as the calibrated magnitudes are listed in Table 10.

The photometric data of the Chandra sources were converted to flux densities based on the zero-point fluxes given in Fukugita et al. (1996) for the $g^{\prime}, r^{\prime}, i^{\prime}, z^{\prime}$ filters, and in Bessell et al. (1998) for the $B, V, R, I$ bands. The bands which are least contaminated by emission lines and that have the closest effective wavelengths to rest-frame $1450 \AA$ were used for obtaining $F_{\lambda}(1450 \AA)$ values for each epoch. Given the fact that we use broadband filters with typical bandwidths of $\sim 1000 \AA$ and that the strongest emission lines in the rest-frame $\sim 1200-1800 \AA$ accessible to us have typical equivalent widths (EWs) of $\lesssim 350 \AA$ (given the redshifts of our sources), the maximum flux contributions of each of these lines to each of our filters should be $\lesssim 35 \%$ (see also, e.g., Elvis et al. 2012). For example, one of the strongest emission lines in the observed-frame optical band, C IV $\lambda 1549$, has EW $\sim 100 \AA$ in PSS 0926+3055 and PSS 1326+0743 (obtained from our ground-based spectroscopy; see below), as well as in Q 0000-263 (see Schneider et al. 1989), hence the contribution

\footnotetext{
23 http://www.sdss.org/dr5/algorithms/sdssUBVRITransform.html

24 http://brucegary.net/dummies/USNO-A2_Method.htm

$25 \mathrm{http}: / /$ www.lco.cl/draft/direct-ccd-users-manual
} 
Table 11

Optical Light Curve and $\alpha_{\mathrm{ox}}$ Data for the Chandra Sources

\begin{tabular}{lccccc}
\hline \hline Quasar & JD & $F_{\lambda}{ }^{\mathrm{a}}$ & $\alpha_{\text {ox }}$ & $\Delta t^{\mathrm{b}}$ & Observatory \\
\hline Q 0000-263 & 2455809.5 & $2.41 \pm 0.04$ & -1.74 & 1.3 & WO \\
& 2456185.5 & $2.35 \pm 0.07$ & -1.76 & 2.4 & WO \\
& 2456186.5 & $2.39 \pm 0.03$ & $\ldots$ & $\ldots$ & WO \\
BR 0351-1034 & 2455624.2 & $0.33 \pm 0.02$ & $\ldots$ & $\ldots$ & WO \\
& 2455626.2 & $0.37 \pm 0.01$ & $\ldots$ & $\ldots$ & WO \\
& 2455831.5 & $0.31 \pm 0.01$ & -1.65 & 0.7 & WO \\
PSS 0926+3055 & 2455864.8 & $0.30 \pm 0.01$ & -1.67 & 0.3 & LCO \\
& 2455625.2 & $2.99 \pm 0.03$ & -1.75 & 0.2 & WO \\
PSS 1326+0743 & 2455962.3 & $2.53 \pm 0.14$ & -1.78 & 4.4 & WO \\
& 2455629.6 & $1.75 \pm 0.03$ & -1.65 & 0.3 & WO \\
& 2455635.5 & $1.74 \pm 0.03$ & $\ldots$ & $\ldots$ & WO \\
& 2456049.3 & $1.58 \pm 0.11$ & -1.64 & 0.3 & WO \\
\hline
\end{tabular}

Notes. For each source, $\alpha_{\mathrm{ox}}$ is given only for the shortest time separations between the optical and Chandra observations.

a Flux density at rest-frame $1450 \AA$ in units of $10^{-16} \mathrm{erg} \mathrm{cm}^{-2} \mathrm{~s}^{-1} \AA^{-1}$.

${ }^{\mathrm{b}}$ Rest-frame days between the optical and Chandra observations.

of this emission line to the flux densities obtained in, e.g., the $i^{\prime}$-band is of the order of $\sim 10 \%$, not considerably larger than the typical photometric uncertainties (see Table 10; there are no published values of emission-line EWs for BR 0351-1034).

The optical fluxes and the corresponding $\alpha_{\text {ox }}$ values are given in Table 11 where we also list the time separations between the optical and Chandra observations that were used for computing $\alpha_{\text {ox }}$. These time separations are on the order of $\approx 1$ day in the rest frame. Based on the photometry in Table 10 and on the results of the Swift sources (Section 3.3.1), we do not consider these time delays to be significant as we do not detect large optical flux variations on such short timescales. However, we do detect optical flux variations at a level of up to $\sim 20 \%$ on significantly longer timescales. Therefore, similar to the Swift sources, we find that $\alpha_{\mathrm{ox}}$ variability is dominated by the X-ray variability, except for PSS $1326+0743$ which is not X-ray variable.

\subsubsection{Optical Spectroscopy}

We searched for optical spectroscopic variations in two of our Chandra sources, which we observed within $\sim 1$ day in the rest frame from their Chandra Cycle 12 observations, by comparing their spectra with archival data. Spectroscopic observations of PSS 0926+3055 and PSS 1326+0743 were performed with the queue-scheduled 9 m Hobby-Eberly Telescope (HET; Ramsey et al. 1998; Shetrone et al. 2007) on 2011 March 1 and 2011 March 10, respectively. Observations of both sources were obtained with the Marcario Low-Resolution Spectrograph (Hill et al. 1998) using the G1 grism with the $3^{\prime \prime}$ slit, providing a resolution of $R \sim 240$, and the OG515 blocking filter. The spectrum of each source was obtained in two sub-exposures of $450 \mathrm{~s}$ each in order to remove cosmic rays. Image reduction and spectral analysis were performed with IRAF using neon and argon wavelength calibration lamps. Flux calibration was performed using a spectrophotometric standard star taken each night and an $I$-band image snapshot that could be compared to SDSS $i$-band magnitudes of nearby field stars. The final, calibrated spectra are shown in Figure 8.

The flux density at rest-frame $1450 \AA$ extracted from our spectrum of PSS $0926+3055$ is a factor of $\sim 2$ larger than the value derived from the SDSS $i$-band magnitude of the source, $i=17.07$, which is only $0.06 \mathrm{mag}$ fainter than the $i^{\prime}$-band magnitude we measure on 2011 March 4, as close as possible to the time of our HET observation. The HET flux density

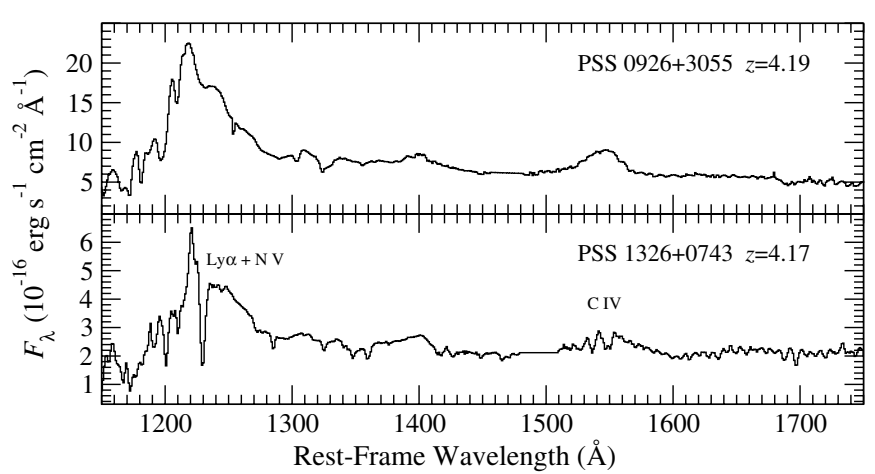

Figure 8. New HET spectra of PSS 0926+3055 (top) and PSS 1326+0743 (bottom). Prominent emission lines are indicated in the bottom panel. The emission line EWs in these sources have not significantly changed compared to the respective archival HET spectra of Vignali et al. (2003).

we measure for this source is also $\sim 60 \%$ larger than the one obtained by Vignali et al. (2003) who used HET spectroscopy with a different standard-star calibration. For PSS 1326+0743, our HET measurement gives a flux density at rest-frame $1450 \AA$ which is only $\sim 5 \%$ larger than the value obtained from its SDSS $i$-band magnitude, $i=17.50$, and is consistent with our $i^{\prime}$-band measurement from 2011 March 14, as close as possible to our HET observation; the value obtained by Vignali et al. (2003) using HET spectroscopy of this source is a factor of $\sim 2$ lower. The large differences in flux density indicate uncertainties on the order of $\approx 0.5-1 \mathrm{mag}$ in the flux calibration between the HET spectra and the photometry as well as between the two pairs of HET spectra. These large uncertainties likely originate from observing a different calibration star at a different position on the sky with respect to the telescope in each epoch as well as from slit losses due to the fact that the HET spectra were not necessarily obtained during photometric conditions. By fitting a power-law continuum and two Gaussians to model the spectral region around the $\mathrm{C}$ IV emission line, we find that the $\mathrm{C}$ IV $\mathrm{EW}$ in both sources agrees to within $\sim 5 \%$ with the values given in Vignali et al. (2003).

\subsection{X-Ray Spectral Variability}

By design, our economical X-ray observations are intended to provide only the minimal number of counts that are sufficient for basic time-series analyses of intensity fluctuations. Naturally, this approach does not allow meaningful X-ray spectral measurements. Table 4 shows that the effective power-law photon indices of our Chandra sources are consistent between Chandra Cycles 12 and 13 and with measurements obtained from highquality X-ray spectroscopy (see Shemmer et al. 2005). Among the Swift sources, only PG 1247+267 and PG 1634+706 have sufficient numbers of counts $(\sim 65-350)$ in three and seven Swift observations, respectively, to perform basic X-ray spectral analysis (see Table 5). We analyzed the spectrum extracted from each of these XRT observations using XSPEC v12 (Arnaud 1996) with a Galactic absorbed power-law model in the observed-frame $0.2-10 \mathrm{keV}$ band. The resultant photon indices were consistent for each source within their errors (at $90 \%$ confidence) among the different epochs. In particular, we do not detect any evidence for steepening in the X-ray spectra of PG 1247+267 and PG $1634+706$ as they become X-ray brighter by factors of $\sim 2$ and $\sim 4$, respectively (cf. Mateos et al. 2007; Sobolewska \& Papadakis 2009; Gibson \& Brandt 2012). We also analyzed the Chandra spectra of PG 1634+706 in the same manner, using the CIAO task PSEXTRACT, and found that the photon index of 
the source in the observed-frame $0.5-8 \mathrm{keV}$ band did not vary significantly (at $90 \%$ confidence) between the different epochs (although most of these are within a few hours in the rest frame from each other).

HS $1700+6416$ has been known to display complex X-ray spectral variability, although the power-law photon index remains constant within the errors (e.g., Lanzuisi et al. 2012). The spectral variability is associated primarily with variations in the intrinsic absorption column density and with potential variable relativistic outflows. Our analysis of the Chandra data of the source is consistent with the Lanzuisi et al. (2012) findings. Unfortunately, the low count rate of the XRT observations prevents us from obtaining additional insight into the nature of these spectral variations.

\section{DISCUSSION}

The basic finding from our exploratory, long-term X-ray monitoring program is that significant $\mathrm{X}$-ray flux variations persist at the highest AGN luminosities and redshifts. In this section, we discuss potential sources for the persistent variability and whether any of these are expected to evolve with cosmic time. We stress again that due to the nature of this pilot project and the small sample size the following discussion is mostly qualitative; a more rigorous treatment is beyond the scope of this work.

\subsection{Were Quasars More X-Ray Variable in the Early Universe?}

Six of our seven luminous RQQs display pronounced X-ray variability similar to many of their nearby and far less-luminous counterparts (e.g., Leighly 1999; Turner et al. 1999; Markowitz \& Edelson 2004; Ponti et al. 2012; Vagnetti et al. 2013). The rest-frame energy band over which the X-ray variability is measured, $\sim 2.6-10.4 \mathrm{keV}$, is also comparable to the restframe energy bands used in surveys of lower-redshift sources. While their variability amplitudes do not exceed those of their lower-luminosity counterparts, our sources do not follow the well-known variability-luminosity anticorrelation observed in the nearby universe (e.g., Lawrence \& Papadakis 1993; P04). This finding is at odds with simplistic light-crossing time considerations since the most luminous sources are expected to host the largest continuum emission regions and thus their light fluctuations are expected to be suppressed.

P04 have found that $\sigma_{\text {rms }}^{2}$ decreases with X-ray luminosity in the observed-frame $0.5-7 \mathrm{keV}$ band $\left(L_{0.5-7}\right)$ up to $L_{0.5-7} \sim 10^{44} \mathrm{erg} \mathrm{s}^{-1}$, and increases slightly beyond that luminosity (see their Figure 12), a result supported by the CDF-S 2 Ms data shown here (Figure 5). This "upturn" in the amplitude-luminosity relation could also be interpreted as an amplitude-redshift relation and an increase in the fraction of variable sources as a function of redshift beyond $z \sim 2$ due to the flux-limited nature of the CDF-S survey. Similar trends have been reported previously by Almaini et al. (2000) and Manners et al. (2002). Vagnetti et al. (2011) report a stronger dependence of X-ray variability on luminosity and a much weaker one on redshift. However, Mateos et al. (2007) do not find a significant dependence between X-ray variability and either luminosity or redshift in the XMM-Newton Lockman Hole survey, Gibson \& Brandt (2012) do not find an increase of X-ray variability with redshift up to $z \sim 4$, and Lanzuisi et al. (2014) do not find an increase in X-ray variability with redshift up to $z \sim 3.5$ in the $X M M-N e w t o n$ COSMOS survey. Although we do not detect an increase in $\sigma_{\text {rms }}^{2}$ with respect to either $L_{0.5-8}$ or redshift be- yond the limits of the $2 \mathrm{Ms}$ CDF-S survey, our variable sources have an average variability amplitude that is similar to the bulk of lower-luminosity sources at $z \lesssim 1$ (Figure 5). This trend is difficult to explain if high-redshift sources follow the same variability-luminosity relation of their lower-redshift counterparts, which is driven primarily by $\mathrm{BH}$ mass (see Section 1). Furthermore, the median of the $\sigma_{\text {rms }}^{2}$ values of our Swift sources, at $\langle z\rangle \simeq 2.0$, is larger than the median of the $\sigma_{\text {rms }}^{2}$ values of our variable Chandra sources at $\langle z\rangle \simeq 4$.2. Notwithstanding the large differences in the sampling patterns between the Chandra and Swift sources, as well as the uncertainties associated with computing $\sigma_{\text {rms }}^{2}$ for individual sources (see Section 3.1), this is an indication that, at least within the limits of our sample, the $\mathrm{X}$-ray variability amplitude does not depend primarily on redshift, and therefore the dependence of X-ray variability on additional parameters should be explored.

\subsection{Black-hole Mass and Accretion Rate Effects}

Since AGN luminosity is not the only parameter controlling the variability amplitude, we must consider the combined effects of the monitoring duration, and the source $\mathrm{BH}$ mass $\left(M_{\mathrm{BH}}\right)$ and accretion rate on our results (e.g., McHardy et al. 2006). Although we detect significant X-ray variability on all restframe timescales $>1$ day at least for the Swift sources, we find that the variability amplitude increases with the temporal baseline up to rest-frame timescales of $\approx 10^{3}$ days. This is in agreement with numerous studies of AGN variability, in the X-rays and in other bands, and is consistent with the generic AGN PSD function where more power is observed at longer timescales (e.g., Fiore et al. 1998; Cid Fernandes et al. 2000; Uttley et al. 2002; Markowitz \& Edelson 2004; Mushotzky et al. 2011). Figure 7 indicates that, within the limits of our sample, the X-ray variability behavior of luminous high-redshift sources is not significantly different from that of nearby AGNs, in terms of their SF (see also Vagnetti et al. 2011). Nevertheless, continued X-ray monitoring is required, especially for the Chandra sources, in order to perform a more detailed comparison between nearby and distant sources. Ideally, what future X-ray missions should provide is a dense and regularly sampled X-ray light curve of luminous, highredshift sources from which high-quality PSD functions can be computed.

Given the fact that AGN variability increases on longer timescales, AGN variability studies based on survey data are expected to show smaller variability amplitudes for higherredshift sources due to cosmic time dilation. Our monitoring project is not subject to such bias since our temporal baseline is not fixed and varies from source to source. The rest-frame temporal baselines for our sources are in the range $\approx 800-3000$ days, comparable to the temporal baselines for the bulk of the sources in the $2 \mathrm{Ms}$ exposure of the CDF-S (Figure 4). Given the negative power-law slope of the PSD function, the variability amplitudes of our sources (and most of the CDF-S sources) are expected to increase as their temporal baselines increase. Our monitoring project should therefore continue in order to allow a much better comparison with the CDF-S sources by utilizing the planned $7 \mathrm{Ms}$ exposure of the CDF-S, thus extending the restframe temporal baselines to $\approx 1000-5000$ days. The continued monitoring will also enable searching for the expected flattening in the PSD function of luminous AGNs.

Since luminosity is a rough proxy to $M_{\mathrm{BH}}$, the amplitudeluminosity anticorrelation may be based on an amplitude$M_{\mathrm{BH}}$ anticorrelation which is due to light-crossing time 
effects (e.g., Lanzuisi et al. 2014). As $M_{\mathrm{BH}}$ increases, the PSD function shifts to lower frequencies, resulting in a decrease in variability amplitude (which is equivalent to integrating the PSD function between two specific frequencies). In addition, the variability amplitude may increase when the normalized accretion rate (in terms of $L / L_{\text {Edd }}$ ) increases (e.g., McHardy et al. 2006, but see also, e.g., González-Martín \& Vaughan 2012; Ponti et al. 2012). Figure 5 may therefore include a combination of different amplitude-luminosity correlations, depending on different values of $L / L_{\text {Edd }}$ or on different accretion modes at different luminosities or redshifts. These correlations may have different slopes and constants in the $\sigma_{\text {rms }}^{2}-L_{0.5-8}$ plane and thus contribute to the upturn and the subsequent flattening in that diagram (Allevato et al. 2010). Testing this possibility requires monitoring of faint AGNs at high redshift $(z \gtrsim 4)$ which is extremely challenging. Such low- $M_{\mathrm{BH}}$ sources may vary even more than the luminous sources in our sample.

According to Papadakis et al. (2008), the upturn and then the flattening observed in the amplitude-luminosity relation in X-ray survey data is due to the fact that as the redshift increases, luminosity increases as well (due to selection effects), but more rapidly than the increase in $M_{\mathrm{BH}}$, resulting in an increase in $L / L_{\text {Edd }}$; the increase in $L / L_{\text {Edd }}$ contributes to the increase in the variability amplitude. As the redshift increases even further, the increase in amplitude must stop due to the effect of cosmic time dilation since the temporal baseline is fixed. In our case, however, the temporal baseline increases continuously, and it is expected that the variability amplitude will continue to increase up to a timescale where the SF flattens. If the trend of having roughly constant $\sigma_{\text {rms }}^{2}$ values as $L_{0.5-8}$ increases is due to a combination of $M_{\mathrm{BH}}$ and $L / L_{\mathrm{Edd}}$ alone, it requires that the decrease in amplitude, due to the increase in $M_{\mathrm{BH}}$, approximately cancels the increase in amplitude as $L / L_{\mathrm{Edd}}$ increases (assuming the temporal baseline is sufficiently long).

We have simulated the combined effects of $M_{\mathrm{BH}}$ and $L / L_{\mathrm{Edd}}$ on $\sigma_{\text {rms }}^{2}$ using the Papadakis et al. (2008) model, and plotted the results for several $L / L_{\text {Edd }}$ values and three different restframe sampling patterns in Figure 5. In short, this model assumes a double power-law PSD function with a constant normalization, ${ }^{26}$ where the power law changes slope from $\alpha=-2$ at the highest frequencies to $\alpha=-1$ at frequencies below a break frequency, $v_{\text {break }}$, where $v_{\text {break }} \propto \eta\left(L / L_{\mathrm{Edd}}\right) M_{\mathrm{BH}}{ }^{-1}$ and $\eta$ is the accretion efficiency, taken here as $\eta=0.1$ (following McHardy et al. 2006). We have integrated the model PSD function in the frequency domain using $8 \mathrm{yr}$ and 1 day for the lowest and highest rest-frame frequencies, respectively, and computed $\sigma_{\text {rms }}^{2}$ as a function of $L_{0.5-8}$, using the Marconi et al. (2004) relation between the X-ray and bolometric luminosities. These frequencies are representative of the total duration and of the minimum sampling period of our Swift sources (considering the binned Swift data for these sources).

Figure 5 demonstrates that using a constant $L / L_{\text {Edd }}=0.5$ for computing $\nu_{\text {break }}$, produces a predicted $\sigma_{\text {rms }}^{2}$ consistent with those of our Swift sources; it also shows that using lower $L / L_{\text {Edd }}$ values result in lower predicted $\sigma_{\mathrm{rms}}^{2}$ values. In fact, $M_{\mathrm{BH}}$ values derived using the single-epoch spectroscopic method based on the $\mathrm{H} \beta$ line for two of our Swift sources, PG 1247+267 and PG $1634+706$, are $1.4 \times 10^{10} M_{\odot}$ and $3.9 \times 10^{10} M_{\odot}$, respectively (with a typical uncertainty of a factor of $\sim 2-3$

\footnotetext{
${ }^{26}$ Ponti et al. (2012) suggest that the normalization of the PSD function
} depends on the accretion rate. on the derived mass), and their respective $L / L_{\text {Edd }}$ values are 0.5 and 0.3 (see Shemmer et al. 2006a, and references therein for more details). These values are consistent with our model $L / L_{\text {Edd }}$ and the derived $M_{\mathrm{BH}}$. Additionally, the McHardy et al. (2006) relation for the break frequency, using the $\mathrm{H} \beta$-based $M_{\mathrm{BH}}$ and $L / L_{\mathrm{Edd}}$ values of PG $1247+267$ and PG $1634+706$, gives $\nu_{\text {break }}=1.04 \times 10^{-7} \mathrm{~Hz}$ and $\nu_{\text {break }}=2.23 \times 10^{-8} \mathrm{~Hz}$, respectively, which are well within the temporal windows of both of these sources. While the $\mathrm{H} \beta$-based $M_{\mathrm{BH}}$ values are among the highest measured for any AGN, the $L / L_{\text {Edd }}$ values of the two Swift sources are typical of quasars and somewhat lower than those of nearby narrow-line Seyfert 1 galaxies. Indeed, this model is also consistent with the $\sigma_{\text {rms }}^{2}$ values of several lowredshift AGNs from the CDF-S survey, which have a roughly similar sampling pattern; such sources, as well as those that lie above this model in Figure 5, may have relatively high accretion rates (although the reliability of single-source measurements must be interpreted with caution; see Allevato et al. 2013).

We ran three additional models of this kind. The first two were designed to match the temporal sampling pattern of the Chandra sources, using $3 \mathrm{yr}$ and 100 days for the lowest and highest restframe frequencies, respectively; one of these uses $L / L_{\text {Edd }}=0.1$ and the other uses $L / L_{\mathrm{Edd}}=0.5$. Figure 5 shows that the model using $L / L_{\text {Edd }}=0.5$ predicts $\sigma_{\text {rms }}^{2}$ values that are consistent with those of two of the variable Chandra sources, Q 0000-263 and PSS $0926+3055$. The third model uses $3 \mathrm{yr}$ and 1 day for the lowest and highest rest-frame frequencies, respectively, and $L / L_{\text {Edd }}=0.1$, mimicking the temporal sampling pattern of a typical variable AGN from the $2 \mathrm{Ms}$ exposure of the CDF-S at $z \sim 1.5$, which is the average redshift of the sources in this survey. This model also appears to predict $\sigma_{\text {rms }}^{2}$ values similar to those observed for several CDF-S sources with assumed $M_{\mathrm{BH}}$ values of $<10^{9} M_{\odot}$. At least to first order, it appears as if the combined effects of $M_{\mathrm{BH}}$ and $L / L_{\mathrm{Edd}}$ can largely explain the observed X-ray variability amplitudes of AGNs over the widest possible ranges of X-ray luminosity and redshift.

\subsection{Additional Contributions to X-Ray Variability in RQQs?}

The source of X-ray variability in AGNs is likely much more complex than the combined effects of $M_{\mathrm{BH}}$ and accretion power on a single source PSD function. For example, spectroscopic observations show that variable obscuration close to the central engine plays a role in the X-ray variability of AGNs (e.g., Risaliti et al. 2009); however, our sources are not expected to be subject to significant obscuration due to their extremely high luminosities (e.g., Hasinger 2008). Except for the X-ray spectral variations detected in HS 1700+6416 (Lanzuisi et al. 2012), we do not detect any major spectral changes in our sources due to the lack of higher-quality multi-epoch spectroscopic observations. Another contribution to X-ray variability may be due to a more distant reflector which can dilute the fluctuations of the more compact and highly variable nuclear X-ray source. While luminous quasars are not expected to exhibit a strong Compton reflection component in their X-ray spectra, due to the diminishing covering factor of a putative torus of gas and dust as AGN luminosity increases (e.g., Ueda et al. 2003; Maiolino et al. 2007; Ricci et al. 2013), two of our sources, PG 1247+267 and PG 1634+706, actually show hints of Compton reflection components (Shemmer et al. 2008, and references therein). Hence it is not clear if and to what extent an X-ray reflector can play a role in the X-ray variability of luminous quasars.

The fact that at least two of our sources, PG 1634+706 and HS 1700+6416, exhibit significant variability on timescales as 
short as a few days in the rest frame is consistent with the idea that the main contribution to the X-ray variability arises from a small region that may not scale with, or have only a weak dependence on, luminosity (or $M_{\mathrm{BH}}$ ). Since $v_{\text {break }}$ of the X-ray PSD function is expected to decrease as $M_{\mathrm{BH}}$ increases, we would have expected much slower variations in our sources with respect to nearby AGNs. However, we do not detect such a trend in our SFs. In particular, based on Figure 7, we do not observe the strong dependence of the SF on luminosity as reported in Vagnetti et al. (2011; cf. their Figure 10), who find that the slope of the SF increases with increasing luminosity; a dependence that is not observed for the PSD function. As we explain above, high $L / L_{\text {Edd }}$ values may compensate for the reduced variability due to the increase in $M_{\mathrm{BH}}$ and contribute to more rapid variability as higher frequencies receive more power.

In addition to the effects of the accretion rate, variable jet emission may contribute to the relatively rapid and significant X-ray variability we observe in our luminous sources. Although our selection of RQQs was intended to minimize such "contamination," RQQs are not necessarily "radio silent" (e.g., Kellermann et al. 1994; Barvainis et al. 1996; Falcke et al. 1996; Blundell \& Beasley 1998; Blundell et al. 2003). However, we do not expect that the relatively weak X-ray emission from the jets in RQQs can dominate the observed X-ray variability (e.g., Miller et al. 2011). In order to quantify, or constrain, the contribution of the jet to X-ray variability in RQQs, and since the radio emission in RQQs may be largely due to star formation (e.g., Padovani et al. 2011), future monitoring campaigns should perform near simultaneous X-ray and radio observations of such sources across a wide luminosity range (e.g., Barvainis et al. 2005).

\section{SUMMARY}

We present initial results from Chandra and Swift monitoring of a sample of seven luminous, RQQs at $1.33 \leqslant z \leqslant 4.35$ extending over $\approx 800-3000$ days in the rest frame. Our X-ray observations are supported by archival X-ray data and by simultaneous or nearly simultaneous optical-UV observations, allowing a qualitative investigation of quasar variability properties at the highest luminosities and redshifts. Our main findings can be summarized as follows:

1. RQQs exhibit an excess in X-ray variability, above the well-known amplitude-luminosity anticorrelation, almost independent of luminosity or redshift, at $L \gtrsim 10^{44} \mathrm{erg} \mathrm{s}^{-1}$ in the $0.5-8 \mathrm{keV}$ band. We suggest that this excess is primarily due to the higher accretion rates at higher luminosities and redshifts.

2. We find no direct evidence of evolution in the X-ray variability of RQQs up to $z \sim 4.2$, but a firmer conclusion will require continued monitoring, especially of our Chandra sources.

3. Our sources vary more on longer timescales than on shorter timescales, in agreement with AGN behavior at lower luminosities or redshifts, and some exhibit significant variability on timescales as short as $\sim 1$ day in the rest frame.

4. We find significant variations in the $\alpha_{\mathrm{ox}}$ values of our Swift sources, implying relative flux variations at a level up to a factor of $\sim 3$, which are dominated by the X-ray variations. We confirm earlier reports that the dispersion in the luminosity-corrected $\alpha_{\text {ox }}$ distribution of type 1 RQQs may be dominated by variability.
5. Except for HS 1700+6416, we do not detect significant $\mathrm{X}$-ray spectral variations in our sources, even for corresponding flux variations of up to a factor of $\sim 4$.

We plan to continue this ongoing study using Chandra in order to characterize better the X-ray variability properties of our four sources at $4.10 \leqslant z \leqslant 4.35$ that currently have only four sparsely sampled X-ray epochs. In particular, our aim is to quantify their variability timescales by means of SFs. We also plan to characterize better the temporal behavior of our three sources at $1.33 \leqslant z \leqslant 2.74$ using Swift. Our results will serve as a benchmark for future X-ray variability studies of distant AGNs, e.g., with the planned CDF-S 7 Ms survey, or with $e R O S I T A$ and its multiepoch, all-sky survey spanning $\gtrsim 4 \mathrm{yr}$.

The scientific results reported in this article are based on observations made by the Chandra X-Ray Observatory and on data obtained from the Chandra Data Archive. Support for this work was provided by the National Aeronautics and Space Administration through Chandra Award Nos. GO1-12132X and GO2-13120X (O.S.) issued by the Chandra X-Ray Observatory Center, which is operated by the Smithsonian Astrophysical Observatory for and on behalf of the National Aeronautics and Space Administration under contract NAS8-03060. We also gratefully acknowledge support from NASA Swift grant NNX08AT26G (O.S.), NASA ADP grant NNX10AC99G (W.N.B.), the Kitzman Fellowship at the Technion (S.K.), and Fondecyt Project 1120328 (P.L.). This work is based, in part, on observations obtained with the Tel Aviv University Wise Observatory $1 \mathrm{~m}$ telescope. The HET is a joint project of the University of Texas at Austin, the Pennsylvania State University, Stanford University, Ludwig-Maximillians-Universität München, and Georg-August-Universität Göttingen. The HET is named in honor of its principal benefactors, William P. Hobby and Robert E. Eberly. We thank Doron Chelouche and Dipankar Maitra for fruitful discussions. This research has made use of the NASA/IPAC Extragalactic Database (NED) which is operated by the Jet Propulsion Laboratory, California Institute of Technology, under contract with the National Aeronautics and Space Administration. This research has also made use of data provided by the High Energy Astrophysics Science Archive Research Center (HEASARC), which is a service of the Astrophysics Science Division at NASA/GSFC and the High Energy Astrophysics Division of the Smithsonian Astrophysical Observatory.

Facilities: CXO (ACIS), Swift (XRT, UVOT)

\section{REFERENCES}

Allevato, V., Paolillo, M., Papadakis, I., \& Pinto, C. 2013, ApJ, 771, 9

Allevato, V., Pinto, C., Paolillo, M., et al. 2010, in AIP Conf. Proc. 1248, X-Ray Astronomy 2009; Present Status, Multi-Wavelength Approach and Future Perspectives, ed. A. Comastri, L. Angelini, \& M. Cappi (Melville, NY: AIP), 491

Almaini, O., Lawrence, A., Shanks, T., et al. 2000, MNRAS, 315, 325

Arnaud, K. A. 1996, in Astronomical Data Analysis Software and Systems V, Conf. Ser. Vol. 101, ed. G. H. Jacoby \& Jeannette Barnes (San Francisco, CA: ASP), 17

Baldwin, J. A., Wampler, E. J., \& Gaskell, C. M. 1989, ApJ, 338, 630

Barr, P., \& Mushotzky, R. F. 1986, Natur, 320, 421

Barvainis, R., Lehár, J., Birkinshaw, M., Falcke, H., \& Blundell, K. M. 2005, ApJ, 618, 108

Barvainis, R., Lonsdale, C., \& Antonucci, R. 1996, AJ, 111, 1431

Bechtold, J., Elvis, M., Fiore, F., et al. 1994, AJ, 108, 374

Bechtold, J., Siemiginowska, A., Shields, J., et al. 2003, ApJ, 588, 119

Becker, R. H., White, R. L., \& Helfand, D. J. 1995, ApJ, 450, 559 
Bentz, M. C., Walsh, J. L., Barth, A. J., et al. 2009, ApJ, 705, 199 Bessell, M. S., Castelli, F., \& Plez, B. 1998, A\&A, 333, 231

Blackburn, J. K. 1995, in ASP Conf. Ser. 77, Astronomical Data Analysis Software and Systems IV, ed. R. A. Shaw, H. E. Payne, \& J. J. E. Hayes (San Francisco, CA: ASP), 367

Blundell, K. M., \& Beasley, A. J. 1998, MNRAS, 299, 165

Blundell, K. M., Beasley, A. J., \& Bicknell, G. V. 2003, ApJL, 591, L103

Burrows, D. N., Hill, J. E., Nousek, J. A., et al. 2005, SSRv, 120, 165

Chartas, G., Kochanek, C. S., Dai, X., et al. 2012, ApJ, 757, 137

Cid Fernandes, R., Sodré, L., Jr, \& Vieira da Silva, L., Jr 2000, ApJ, 544, 123

Collier, S. J., Horne, K., Kaspi, S., et al. 1998, ApJ, 500, 162

Condon, J. J., Cotton, W. D., Greisen, E. W., et al. 1998, AJ, 115, 1693

Dickey, J. M., \& Lockman, F. J. 1990, ARA\&A, 28, 215

Djorgovski, S. G., Gal, R. R., Odewahn, S. C., et al. 1998, in Wide Field Surveys in Cosmology, 14th IAP Meeting (Paris: Editions Frontieres), 89

Eadie, W. T., Dryard, D., James, F. E., Roos, M., \& Sadoulet, B. 1971, Statistical Methods in Experimental Physics (Amsterdam: North-Holland)

Elvis, M., Hao, H., Civano, F., et al. 2012, ApJ, 759, 6

Emmanoulopoulos, D., McHardy, I. M., \& Uttley, P. 2010, MNRAS, 404, 931

Falcke, H., Sherwood, W., \& Patnaik, A. R. 1996, ApJ, 471, 106

Ferrero, E., \& Brinkmann, W. 2003, A\&A, 402, 465

Fiore, F., Laor, A., Elvis, M., Nicastro, F., \& Giallongo, E. 1998, ApJ, 503, 607

Freeman, P. E., Kashyap, V., Rosner, R., \& Lamb, D. Q. 2002, ApJS, 138, 185

Fukugita, M., Ichikawa, T., Gunn, J. E., et al. 1996, AJ, 111, 1748

Garmire, G. P., Bautz, M. W., Ford, P. G., Nousek, J. A., \& Ricker, G. R. 2003, Proc. SPIE, 4851, 28

Gehrels, N. 1986, ApJ, 303, 336

Gehrels, N., Chincarini, G., Giommi, P., et al. 2004, ApJ, 611, 1005

George, I. M., Turner, T. J., Yaqoob, T., et al. 2000, ApJ, 531, 52

Gibson, R. R., \& Brandt, W. N. 2012, ApJ, 746, 54

Gibson, R. R., Brandt, W. N., \& Schneider, D. P. 2008, ApJ, 685, 773

González-Martín, O., \& Vaughan, S. 2012, A\&A, 544, A80

Green, A. R., McHardy, I. M., \& Lehto, H. J. 1993, MNRAS, 265, 664

Green, R. F., Pier, J. R., Schmidt, M., et al. 1980, ApJ, 239, 483

Grupe, D., Mathur, S., Wilkes, B., \& Elvis, M. 2004, AJ, 127, 1

Grupe, D., Mathur, S., Wilkes, B., \& Osmer, P. 2006, AJ, 131, 55

Haardt, F., \& Maraschi, L. 1991, ApJL, 380, L51

Hasinger, G. 2008, A\&A, 490, 905

Hill, G. J., Nicklas, H. E., MacQueen, P. J., et al. 1998, Proc. SPIE, 3355, 375

Hughes, P. A., Aller, H. D., \& Aller, M. F. 1992, ApJ, 396, 469

Irwin, M., McMahon, R. G., \& Hazard, C. 1991, in ASP Conf. Ser. 21, The Space Distribution of Quasars, ed. D. Crampton (San Francisco, CA: ASP), 67

Isaak, K. G., McMahon, R. G., Hills, R. E., \& Withington, S. 1994, MNRAS, 269, L28

Jansen, F., Lumb, D., Altieri, B., et al. 2001, A\&A, 365, L1

Jester, S., Schneider, D. P., Richards, G. T., et al. 2005, AJ, 130, 873

Just, D. W., Brandt, W. N., Shemmer, O., et al. 2007, ApJ, 665, 1004

Kaspi, S., Brandt, W. N., Maoz, D., et al. 2007, ApJ, 659, 997

Kaspi, S., Brandt, W. N., \& Schneider, D. P. 2000a, AJ, 119, 2031

Kaspi, S., Smith, P. S., Netzer, H., et al. 2000b, ApJ, 533, 631

Kellermann, K. I., Sramek, R., Schmidt, M., Shaffer, D. B., \& Green, R. 1989, AJ, 98, 1195

Kellermann, K. I., Sramek, R. A., Schmidt, M., Green, R. F., \& Shaffer, D. B. 1994, AJ, 108, 1163

Kelly, B. C., Bechtold, J., Siemiginowska, A., Aldcroft, T., \& Sobolewska, M. 2007, ApJ, 657, 116

Kirsch, M. G., Briel, U. G., Burrows, D., et al. 2005, Proc. SPIE, 5898, 22

Kraft, R. P., Burrows, D. N., \& Nousek, J. A. 1991, ApJ, 374, 344

Lanzuisi, G., Giustini, M., Cappi, M., et al. 2012, A\&A, 544, A2

Lanzuisi, G., Ponti, G., Salvato, M., et al. 2014, ApJ, 781, 105

Lawrence, A., \& Papadakis, I. 1993, ApJL, 414, L85

Leighly, K. M. 1999, ApJS, 125, 297

Lu, Y., \& Yu, Q. 2001, MNRAS, 324, 653

Luo, B., Bauer, F. E., Brandt, W. N., et al. 2008, ApJS, 179, 19

Lyons, L. 1991, Data Analysis for Physical Science Students (Cambridge: Cambridge Univ. Press)

Maiolino, R., Shemmer, O., Imanishi, M., et al. 2007, A\&A, 468, 979

Manners, J., Almaini, O., \& Lawrence, A. 2002, MNRAS, 330, 390

Marconi, A., Risaliti, G., Gilli, R., et al. 2004, MNRAS, 351, 169

Markowitz, A., \& Edelson, R. 2004, ApJ, 617, 939

Markowitz, A., Edelson, R., Vaughan, S., et al. 2003, ApJ, 593, 96

Mateos, S., Barcons, X., Carrera, F. J., et al. 2007, A\&A, 473, 105

McHardy, I. M., Koerding, E., Knigge, C., Uttley, P., \& Fender, R. P. 2006, Natur, 444,730
Miller, B. P., Brandt, W. N., Schneider, D. P., et al. 2011, ApJ, 726, 20

Misawa, T., Eracleous, M., Chartas, G., \& Charlton, J. C. 2008, ApJ, 677, 863

Mushotzky, R. F., Edelson, R., Baumgartner, W., \& Gandhi, P. 2011, ApJL, 743, L12

Nandra, K., George, I. M., Mushotzky, R. F., Turner, T. J., \& Yaqoob, T. 1997, ApJ, 476, 70

Netzer, H., Heller, A., Loinger, F., et al. 1996, MNRAS, 279, 429

Neugebauer, G., Green, R. F., Matthews, K., et al. 1987, ApJS, 63, 615

O’Neill, P. M., Nandra, K., Papadakis, I. E., \& Turner, T. J. 2005, MNRAS, 358, 1405

Padovani, P., Miller, N., Kellermann, K. I., et al. 2011, ApJ, 740, 20

Page, K. L., Reeves, J. N., O’Brien, P. T., Turner, M. J. L., \& Worrall, D. M. 2004, MNRAS, 353, 133

Paolillo, M., Schreier, E. J., Giacconi, R., Koekemoer, A. M., \& Grogin, N. A. 2004, ApJ, 611, 93 (P04)

Papadakis, I. E. 2004, MNRAS, 348, 207

Papadakis, I. E., Chatzopoulos, E., Athanasiadis, D., Markowitz, A., \& Georgantopoulos, I. 2008, A\&A, 487, 475

Piconcelli, E., Jimenez-Bailón, E., Guainazzi, M., et al. 2005, A\&A, 432, 15

Ponti, G., Papadakis, I., Bianchi, S., et al. 2012, A\&A, 542, A83

Ramsey, L. W., Adams, M. T., Barnes, T. G., et al. 1998, Proc. SPIE, 3352,34

Reimers, D., Clavel, J., Groote, D., et al. 1989, A\&A, 218, 71

Reimers, D., Hagen, H.-J., Schramm, J., Kriss, G. A., \& Shull, J. M. 2005, A\&A, 436, 465

Ricci, C., Paltani, S., Awaki, H., et al. 2013, A\&A, 553, A29

Risaliti, G., Miniutti, G., Elvis, M., et al. 2009, ApJ, 696, 160

Roming, P. W. A., Kennedy, T. E., Mason, K. O., et al. 2005, SSRv, 120, 95

Saez, C., Brandt, W. N., Gallagher, S. C., Bauer, F. E., \& Garmire, G. P. 2012, ApJ, 759, 42

Schmidt, M., \& Green, R. F. 1983, ApJ, 269, 352

Schneider, D. P., Hall, P. B., Richards, G. T., et al. 2007, AJ, 134, 102

Schneider, D. P., Richards, G. T., Hall, P. B., et al. 2010, AJ, 139, 2360

Schneider, D. P., Schmidt, M., \& Gunn, J. E. 1989, AJ, 98, 1507

Shemmer, O., Brandt, W. N., Netzer, H., Maiolino, R., \& Kaspi, S. 2006a, ApJL, 646, L29

Shemmer, O., Brandt, W. N., Netzer, H., Maiolino, R., \& Kaspi, S. 2008, ApJ, 682,81

Shemmer, O., Brandt, W. N., Schneider, D. P., et al. 2006b, ApJ, 644, 86

Shemmer, O., Brandt, W. N., Vignali, C., et al. 2005, ApJ, 630, 729

Shemmer, O., Romano, P., Bertram, R., et al. 2001, ApJ, 561, 162

Shetrone, M., Cornell, M. E., Fowler, J. R., et al. 2007, PASP, 119, 556

Snowden, S. L. 2002, arXiv:astro-ph/0203311

Sobolewska, M. A., \& Papadakis, I. E. 2009, MNRAS, 399, 1597

Spergel, D. N., Bean, R., Doré, O., et al. 2007, ApJS, 170, 377

Steffen, A. T., Strateva, I., Brandt, W. N., et al. 2006, AJ, 131, 2826

Storrie-Lombardi, L. J., McMahon, R. G., Irwin, M. J., \& Hazard, C. 1996, ApJ, 468, 121

Tananbaum, H., Avni, Y., Green, R. F., Schmidt, M., \& Zamorani, G. 1986, ApJ, 305,57

Townsley, L. K., Broos, P. S., Garmire, G. P., \& Nousek, J. A. 2000, ApJL, 534, L139

Trevese, D., Paris, D., Stirpe, G. M., Vagnetti, F., \& Zitelli, V. 2007, A\&A, 470,491

Truemper, J. 1982, AdSpR, 2, 241

Turner, T. J., George, I. M., Nandra, K., \& Turcan, D. 1999, ApJ, 524, 667

Ueda, Y., Akiyama, M., Ohta, K., \& Miyaji, T. 2003, ApJ, 598, 886

Ulrich, M.-H., Maraschi, L., \& Urry, C. M. 1997, ARA\&A, 35, 445

Uttley, P., \& Mchardy, I. M. 2004, PThPS, 155, 170

Uttley, P., McHardy, I. M., \& Papadakis, I. E. 2002, MNRAS, 332, 231

Vagnetti, F., Antonucci, M., \& Trevese, D. 2013, A\&A, 550, A71

Vagnetti, F., Turriziani, S., \& Trevese, D. 2011, A\&A, 536, A84

Vagnetti, F., Turriziani, S., Trevese, D., \& Antonucci, M. 2010, A\&A, 519, A17

Vanden Berk, D. E., Richards, G. T., Bauer, A., et al. 2001, AJ, 122, 549

Vaughan, S., Edelson, R., Warwick, R. S., \& Uttley, P. 2003, MNRAS, 345,1271

Vignali, C., Brandt, W. N., Schneider, D. P., Garmire, G. P., \& Kaspi, S. 2003, AJ, 125,418

Vignali, C., Brandt, W. N., Schneider, D. P., \& Kaspi, S. 2005, AJ, 129, 2519

Webb, J. K., Parnell, H. C., Carswell, R. F., McMahon, R. G., \& Irwin, M. J. 1988, Msngr, 51, 15

Weisskopf, M. C., Tananbaum, H. D., Van Speybroeck, L. P., \& O’Dell, S. L. 2000, Proc. SPIE, 4012, 2

Xue, Y. Q., Luo, B., Brandt, W. N., et al. 2011, ApJS, 195, 10

York, D. G., Adelman, J., Anderson, J. E., Jr., et al. 2000, AJ, 120, 1579 